\title{
Statistical Multi-Criteria Evaluation of Non-Nuclear Asteroid Deflection Methods
}

\author{
Nicolas Thiry, Massimiliano Vasile \\ Aerospace Centre of Excellence, Department of Mechanical \&3 Aerospace Engineering, \\ University of Strathclyde
}

\begin{abstract}
In this paper we assess and compare the effectiveness of four classes of nonnuclear asteroid deflection methods applied to a wide range of virtual collision scenarios. We consider the kinetic impactor, laser ablation, the ion beaming technique and two variants of the gravity tractor. A simple but realistic model of each deflection method was integrated within a systematic approach to size the spacecraft and predict the achievable deflection for a given mission and a given maximum mass at launch. A sample of 100 synthetic asteroids was then created from the current distribution of NEAs and global optimisation methods were used to identify the optimal solution in each case according to two criteria: the minimum duration between the departure date and the time of virtual impact required to deflect the NEA by more than two Earth radii and the maximum miss-distance achieved within a total duration of 10 years. Our results provide an interesting insight into the range of applicability of individual deflection methods and argue the need to develop multiple methods in parallel for a global mitigation of all possible threats.
\end{abstract}

Keywords: Asteroid deflection, laser ablation, kinetic impactor, ion beam shepherd, gravity tractor

\section{Introduction}

Near Earth Asteroids (NEA) are defined as asteroids with perihelia lower than 1.3 astronomical units (AU). Potentially hazardous asteroids (PHA)

Email address: massimiliano.vasile@strath.ac.uk (Massimiliano Vasile) 
represent a portion of the NEAs whose current orbits has a Minimum Orbit Interception Distance (MOID) with the Earth's orbit which is less than 0.05 $\mathrm{AU}$ and whose diameter is at least $100 \mathrm{~m}$. PHAs are deemed to represent a risk as they could come into a collision course with the Earth due to perturbations affecting their orbits (Chapman, 2004).

Several deflection methods have been proposed over the years to mitigate the risk of an impact of a PHA with the Earth. Most of the strategies proposed fall into two categories: impulsive and slow-push. Impulsive strategies are usually modelled with an instantaneous change of momentum given by, for example, a nuclear explosion (nuclear interceptor) or the hypervelocity impact of a spacecraft (kinetic impactor) with the asteroid. Slow-push methods, on the other hand, allow for a more controllable deflection manoeuvre by exerting a small continuous and controllable force on the asteroid over an extended period of time. In Sanchez et al. (2009), the authors proposed a comparative analysis of several deflection methods considering thousands of mission scenarios and a number of representative PHAs.

Following the same idea, this paper proposes a new comparative assessment of four classes of asteroid deflection methods for a wide range of collision scenarios. The classes selected for this comparison are: the kinetic impactor (Tedeschi et al. (1995)), the laser ablation (Phipps et al. (1996)), the ion beaming technique (Bombardelli et al. (2013)) and the gravity tractor ( $\mathrm{Lu}$ and Love1 (2005)). For the kinetic impactor we will put to the test the simplest variant with highest technology readiness level but will discuss the potentiality of a version using low-thrust transfers introduced by Conway (1997). For the gravity tractor we will analyse two different configurations.

The laser ablation and the ion beaming were not part of the methods analysed by Sanchez et al. (2009). Furthermore, in this paper, a sample of 100 synthetic PHAs are created from the current distribution of known NEAs and used to build a set of mission scenarios for each deflection method. In all cases, the argument of perigee of the orbit of the PHA is modified so that the virtual asteroid crosses the ecliptic plane at a distance of $1 \mathrm{AU}$ from the Sun. A fixed asteroid mass of $4 \times 10^{9} \mathrm{~kg}$ is considered throughout this study (unless otherwise stated), which corresponds to an estimated diameter of 156 identical the size of asteroid 2011AG5 which was previously considered by NEOSHIELD (2012) and is also comparable to the size of Didymoon which will be the target of the AIDA demonstrator mission.

The methodology in this paper also differs from Sanchez et al. (2009) in that the deflection models are integrated with a revised system sizing 
approach to quantify the mass of the spacecraft at launch and predict the achievable deflection for a given epoch. Furthermore it is assumed that all methods fully exploit the maximum interplanetary launch capability of $10 \mathrm{mt}$ (for a $c_{3}=0 \mathrm{~km}^{2} / \mathrm{s}^{2}$ ), equivalent to that of Delta 4 Heavy RS-68A upgrade version.

The available system mass after the transfer to the target asteroid is used to evaluate the achievable deflection. For the case of the kinetic impactor, a direct injection using a multiple-revolution Lambert arc is considered. For the case of slow-push methods, a low-thrust transfer is retained in order to take advantage of the large electrical power available which would otherwise remain unused during the transfer phase.

A single objective global optimisation technique is then used to find an optimal solution for each scenario within a limited mission duration. A memetic multi-objective optimiser is then also used to identify solutions that are optimal with respect to two criteria: the minimum duration between the departure date and the time of virtual impact required to deflect the PHA by more than 2 Earth radii or the miss-distance achieved within a maximum duration of 10 years.

\section{Fundamentals of Deflection Astrodynamics}

In this section we briefly recall the formulas to calculate the deflection and the associated impact parameter given either an impulsive or a slowpush deflection action. A more extensive treatment can be found in Vasile and Colombo (2008); Colombo et al. (2009).

\subsection{Impulsive Deflection}

The effect of an impulsive change in the velocity of the asteroid induces a variation of its orbit and related orbital elements. The assumption is that this variation is small compared to the asteroid-Sun distance, thus the modified orbit remains in close proximity to the undeflected one. In this case, given the instantaneous change in the asteroid velocity vector $\delta \mathbf{v}=\left[\delta v_{t}, \delta v_{n}, \delta v_{h}\right]^{T}$ in a tangential, normal, out-of-plane reference frame, the position of the deflected asteroid with respect to the undeflected one at true anomaly $\theta_{\text {MoID }}$ along the 
orbit of the undeflected asteroid is:

$$
\begin{aligned}
\delta x_{r}= & \frac{r}{a} \delta a+\frac{a e \sin \theta_{\mathrm{MOID}}}{\sqrt{1-e^{2}}} \delta M-a \cos \theta_{\mathrm{MOID}} \delta e \\
\delta y_{\theta}= & \frac{r}{\left(1-e^{2}\right)^{3 / 2}}\left(1+e \cos \theta_{\mathrm{MOID}}\right)^{2} \delta M+r \delta \omega \\
& +\frac{r \sin \theta_{\mathrm{MOID}}}{\left(1-e^{2}\right)}\left(2+e \cos \theta_{\mathrm{MOID}}\right) \delta e+r \cos i \delta \Omega \\
\delta z_{h}= & r\left(\sin \theta_{\mathrm{MOID}}^{*} \delta i-\cos \theta_{\mathrm{MOID}}^{*} \sin i \delta \Omega\right)
\end{aligned}
$$

where $\delta \mathbf{r}=\left[\delta x_{r}, \delta y_{\theta}, \delta z_{h}\right]^{T}$ is the displacement vector in a radial, transversal, out-of-plane reference frame attached to the undeflected asteroid, $\theta_{\text {MoID }}$ is the true anomaly of the point of Minimum Orbit Intersection Distance (MOID), $\theta_{\text {MOID }}^{*}=\theta_{\text {MOID }}+\omega, r, a, e, i$ and $\omega$ are respectively the radius, semi-major axis, eccentricity, inclination, argument of the pricentre of the orbit of the undeflected asteroid, and $\delta a, \delta e, \delta i, \delta \Omega, \delta \omega, \delta M$ are the variations of the orbital parameters due to $\delta \mathbf{v}$. The variation of the orbital elements are given by:

$$
\begin{aligned}
\delta a & =\frac{2 a^{2} V}{\mu} \delta v_{t} \\
\delta e & =\frac{1}{V}\left(2\left(e+\cos \theta_{d}\right) \delta v_{t}-\frac{r}{a} \sin \theta_{d} \delta v_{h}\right) \\
\delta I & =\frac{r \cos \varpi_{d}}{h} \delta v_{h} \\
\delta \Omega & =\frac{r \sin \varpi_{d}}{h \sin I} \delta v_{h} \\
\delta \omega & =\frac{1}{e V}\left(2 \sin \theta_{d} \delta v_{t}+\left(2 e+\frac{r}{a} \cos \theta_{d}\right) \delta v_{n}\right)-\frac{r \sin \varpi_{d} \cos I}{h \sin I} \delta v_{h} \\
\delta M & =\delta M_{n}-\frac{b}{e a V}\left(2\left(1+\frac{e^{2} r}{p}\right) \sin \theta_{d} \delta v_{t}+\frac{r}{a} \cos \theta_{d} \delta v_{n}\right)
\end{aligned}
$$

where $\theta_{d}$ is the true anomaly at the deflection epoch, $\varpi_{d}=\theta_{d}+\omega$ is the argument of latitude $p=a\left(1-e^{2}\right)$ is the semilatus rectum, $h=\sqrt{\mu a\left(1-e^{2}\right)}$ is the angular momentum, $r=p /(1+e \cos \theta)$ is the orbital radius, and $V$ is the instantaneous asteroid velocity modulus. The time dependent variation of the mean anomaly $\delta M_{n}$ is given by:

$$
\delta M_{n}=\frac{3}{2} \frac{\sqrt{\mu}}{a^{5 / 2}} \Delta t \delta a
$$


From the deflection $\delta \mathbf{r}$ the impact parameter $b$ on the impact plane at the time of the MOID can be computed. The impact plane can be defined as the plane centered in the Earth and perpendicular to the velocity vector of the undeviated asteroid with respect to the Earth, $\mathbf{U}_{\mathrm{NEO}}$, at the time of the impact (see Fig. 1 where $\mathbf{v}_{E}$ is the velocity of the Earth). The simplifying assumption is that the velocity vector of the deflected asteroid remains parallel to the one of the undeflected asteroid at the MOID. The deflection vector $\mathbf{x}_{b}$ in the b-plane coordinates can be expressed as:

$$
\mathbf{x}_{b}\left(t_{\mathrm{MOID}}\right)=\left[\begin{array}{lll}
\xi & \eta & \zeta
\end{array}\right]^{T}=\left[\begin{array}{lll}
\widehat{\xi} & \widehat{\eta} & \widehat{\zeta}
\end{array}\right]^{T} \delta \mathbf{r}=\mathbf{B} \delta \mathbf{r}
$$

where

$$
\widehat{\eta}=\frac{\mathbf{U}_{N E O}}{U_{N E O}}, \quad \hat{\xi}=\frac{\mathbf{v}_{E} \wedge \widehat{\eta}}{\left\|\mathbf{v}_{E} \wedge \widehat{\eta}\right\|}, \quad \hat{\zeta}=\widehat{\xi} \wedge \widehat{\eta}
$$

If one then calls $\delta æ=[\delta a, \delta e, \delta I, \delta \omega, \delta \Omega, \delta M]^{T}$ the vector of the variations of the parameters, and $\mathbf{A}$ and $\mathbf{G}$ the two matrices such that $\delta æ=\mathbf{G} \delta \mathbf{v}$ and $\delta \mathbf{r}\left(t_{\mathrm{MOID}}\right)=\mathbf{A} \delta \rightsquigarrow$ then we have:

$$
\mathbf{x}_{b}\left(t_{\text {MOID }}\right)=\mathbf{B A G} \delta \mathbf{v}
$$

with the impact parameter $b$ :

$$
b=\sqrt{\xi^{2}+\zeta^{2}}
$$

Note that other deflection formulas can be derived from Eq. (8) by assuming for example that the deflection is not introducing any geometric variation on the b-plane but only a temporal variation $\delta M_{n}$. However, retaining only the temporal variation precludes the possibility to study deflection actions with short warning times or leading to relatively small variations of the semimajor axis. Furthermore, when the asteroid's orbit is tangent to the one of the Earth and the undeflected asteroid is due to collide, a change in the orbital period would result in a zero variation of the $b$ parameter although a collision is temporary avoided. This highlights the importance of all the components of the $\mathbf{x}_{b}$ vector as explained in Vasile and Colombo (2008).

\subsection{Slow-Push Deflection}

For the slow-push deflection we follow a treatment similar to the one proposed in Colombo et al. (2009). The variation of the orbital parameters 

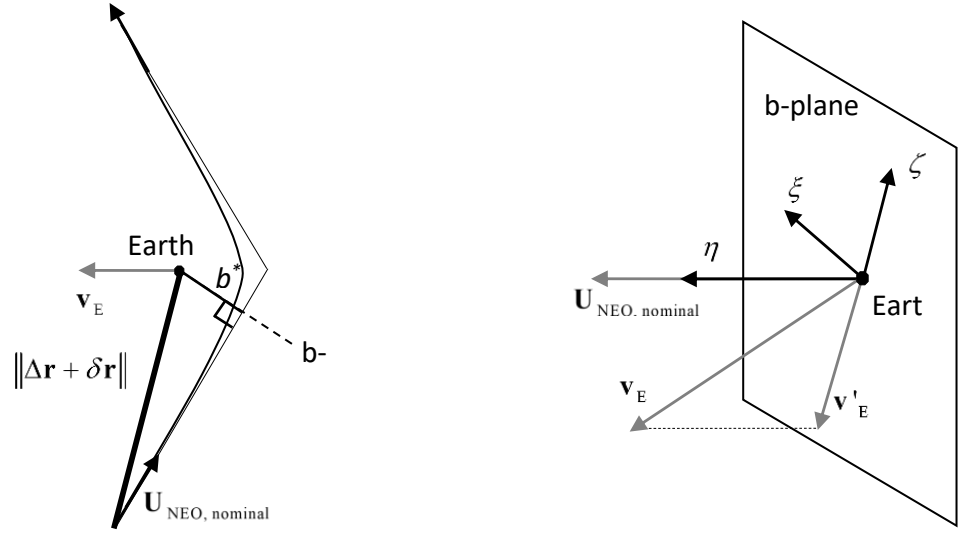

Figure 1: The b-plane and the impact parameter b

is calculated by integration of the following Gauss' planetary equations in non-singular elements from the time $t_{d}$ when the deflection action $\mathbf{u}$ starts 
until the time $t_{e}$ when the deflection action stops:

$$
\begin{aligned}
\frac{d a}{d L}= & \frac{2 a^{3} B^{2}}{\mu}\left[\frac{\left(P_{2} \sin L-P_{1} \cos L\right)}{\Phi^{2}(L)} u_{r}+\frac{1}{\Phi(L)} u_{t}\right] \\
\frac{d P_{1}}{d L}= & \frac{B^{4} a^{2}}{\mu}\left\{\left[-\frac{\cos L}{\Phi^{2}(L)} u_{r}+\left(\frac{P_{1}+\sin L}{\Phi^{3}(L)}+\frac{\sin L}{\Phi^{2}(L)}\right) u_{t}\right]+\right. \\
& \left.-P_{2} \frac{Q_{1} \cos L-Q_{2} \sin L}{\Phi^{3}(L)} u_{h}\right\} \\
\frac{d P_{2}}{d L}= & \frac{B^{4} a^{2}}{\mu}\left\{\left[\frac{\sin L}{\Phi^{2}(L)} u_{r}+\left(\frac{P_{2}+\cos L}{\Phi^{3}(L)}+\frac{\cos L}{\Phi^{2}(L)}\right) u_{t}\right]+\right. \\
\frac{d Q_{1}}{d L}= & \frac{B^{4} a^{2}}{2 \mu}\left(1+Q_{1}^{2}+Q_{2}^{2}\right) \frac{\sin L}{\Phi^{3}(L)} u_{h} \\
\frac{d Q_{2}}{d L}= & \frac{B^{4} a^{2}}{2 \mu}\left(1+Q_{1}^{2} \sin L\right. \\
\Phi_{h}(L) & \frac{\cos L}{\Phi^{3}(L)} u_{h} \\
\frac{d t}{d L}= & \sqrt{\frac{a^{3}}{\mu} \frac{B^{3}}{\Phi^{2}(L)}}
\end{aligned}
$$

where $L$ is the true longitude, $B=\sqrt{1+P_{1}^{2}+P_{2}^{2}}$ and $\Phi(L)=1+P_{1} \sin L+$ $P_{2} \cos L$. The integration can be performed analytically, using the asymptotic expansions in Zuiani et al. (2012), or numerically, and then converted into the corresponding variation of the orbital elements. Note that when the integration is numerical it is more convenient to use the variation of the elements in time, while the analytical integration is in the true longitude. For further details, please refer to Zuiani and Vasile (2015). Equations (1) and (4) are then applied to give:

$$
\mathbf{x}_{b}\left(t_{\mathrm{MOID}}\right)=\mathbf{B A} \int_{t_{d}}^{t_{e}} \mathbf{G u} d t
$$

It is worth recalling that the variation of the mean anomaly in this case is given by:

$$
\delta M=\left(n_{e}-n\right) t_{\mathrm{MOID}}+n t_{d}-n_{e} t_{e}+\Delta M
$$

where $\Delta M$ is the time integral of the geometric part and

$$
n_{e}=\sqrt{\frac{\mu}{(a+\Delta a)^{3}}}
$$




\section{Deflection Methods}

In this section we revise some existing models of deflection actions and their variants and introduce a discussion on their applicability and effectiveness. The discussion will allow us to short-list only four methods that will then be applied to the selected sample of virtual impactors.

\subsection{Kinetic Impactor}

The idea of the kinetic impactor is to impart a slight alteration in the velocity of an asteroid by colliding a spacecraft into it at high speed. The simplest version of this deflection concept assumes a direct injection (single impulse) into an interception trajectory from the Earth to the asteroid. In this paper the trajectory is calculated as the solution of a multi-revolution Lambert arc. Therefore, the mass $m_{s / c}$ of the spacecraft and its relative velocity $\delta v_{s / c}$ at the deflection date $t_{d}$ are a function of both the time of flight ToF and departure date $t_{D}$ from the Earth as well as the interplanetary injection capability of the launcher. Figure 2 shows the launch capability of

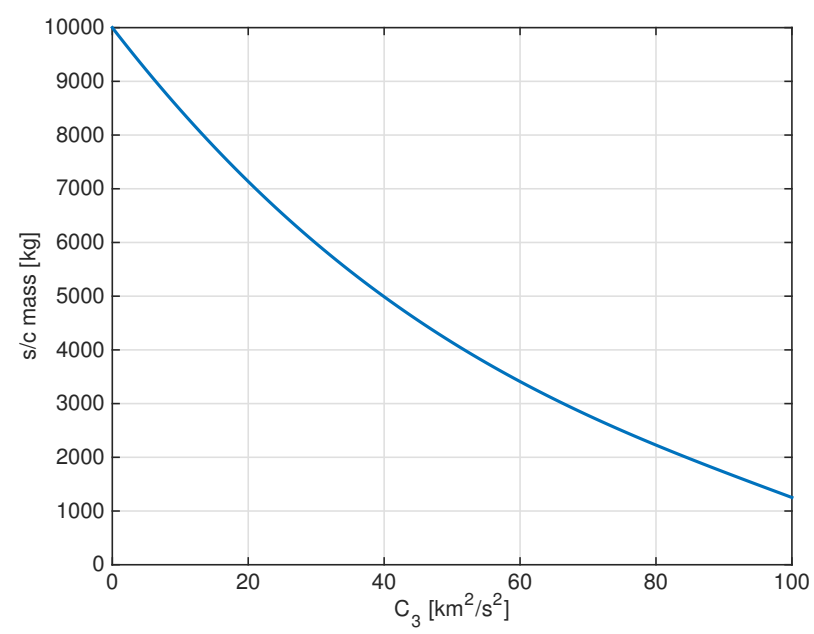

Figure 2: Spacecraft mass $m_{s / c}$ as a function of the $c_{3}$ escape energy from the regression laws of Wise et al. (2010) for the Delta IV Heavy - RS-68A upgrade version

the Delta 4 Heavy RS-68A upgrade version, considered throughout this study, as a function of the $c_{3}$ escape energy $\left(\right.$ in $\left.\mathrm{km}^{2} / \mathrm{s}^{2}\right)$. 
The variation of velocity imparted by the spacecraft to the asteroid is then computed with a simple conservation of momentum equation, assuming a momentum enhancement factor $\beta=1$ :

$$
\delta v=\beta \frac{m_{s / c}}{m_{a}} \delta v_{s / c}
$$

Once the variation of the velocity is available, formulae (1) to (8) can be used to compute the impact parameter. As an illustrative example, Figure 3 shows the achieved impact parameter $b$ as a function of departure date and time of flight considering a kinetic impactor injected into a transfer orbit by a Delta 4 Heavy rocket to a virtual version of $2011 \mathrm{AG} 5^{1}$.

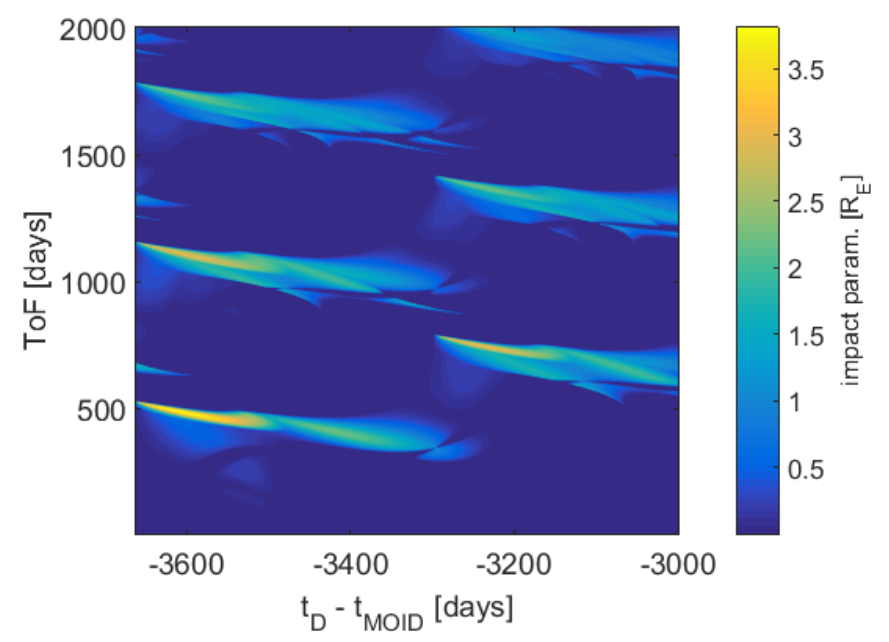

Figure 3: Impact parameter as a function of the departure date $t_{D}$ and time of flight $T o F$ for 2011AG5

\subsection{Ion Beaming}

Ion beaming was proposed by Bombardelli et al. (2013) as a technique to deflect asteroid with the name Ion Beam Shepherd (IBS). The idea is to use an ion engine to transfer momentum to the asteroid by beaming a flow of ions. In order to maintain the relative position between the deflecting spacecraft and the asteroid, a second engine needs to be positioned on the opposite

\footnotetext{
${ }^{1}$ See section 5 for more details on our virtual asteroid models
} 
side of the spacecraft to balance the thrust coming from the engine beaming the ions on the PHA. In the following we assume a momentum transfer efficiency of 1, which means that all the ions are impinging the asteroid, negligible gravity tugging effect at the operation distance (less than $10 \mathrm{mN}$ for distances larger than $500 \mathrm{~m}$ ) and the same thrust for the two engines. The acceleration imparted onto the asteroid is then plugged into equations (7) to get the resulting deflection.

A low-thrust transfer is considered in the case of this method, which allows using the electric propulsion system also during the transfer phase. The spherical shaping method introduced in the work of Novak and Vasile (2011) was used to compute low-cost, low-thrust trajectories as a function of the ToF and departure epoch for the the mission. An example of calculated trajectory to asteroid 2011AG5, requiring a $\Delta v$ of $7.8 \mathrm{~km} / \mathrm{s}$ is shown in Fig. 4.

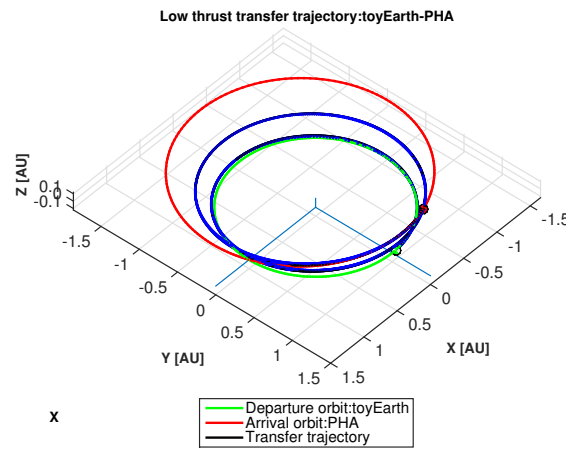

(a)

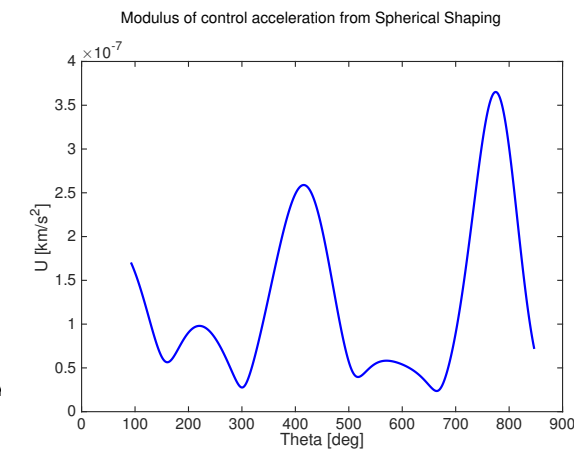

(b)

Figure 4: Example of calculated low thrust transfer trajectory and modulus of the control acceleration with the spherical shaping method for $T o F=847$ days and $t_{D}=-3615$ days before virtual impact with 2011AG5

Considering an $I_{s p}$ of $3000 \mathrm{~s}$ and given a departure mass, our algorithm returns the mass at arrival but also the maximum thrust and power required to realise this transfer. From this information, the mass required for the EPS, power systems and radiators can be computed, assuming Solar Electric Propulsion as a primary power source (SEP) and an oversising coefficient that can vary between 1 and 10. Oversising means that we consider a higher propulsion capability than what is required for the transfer phase. It allows one to increase the thrust that can be generated during the later deflection 
phase but also penalises the amount of propellant mass that will be available during the deflection phase since more mass needs to be allocated to the different subsystems.

Efficient (30\% from solar to electrical power) triple junction solar arrays are assumed in our study. In line with the predicted performance of Orbital ATK's UltraFlex and Megaflex arrays, we consider a specific array mass of $10 \mathrm{~kg} / \mathrm{kW}$ throughout this study, scaling with the power required at 1 AU. An additional $5 \mathrm{~kg} / \mathrm{kW}$, scaling with the peak power at perihelion, models the other components of the power subsystem, including PCDU.

Typically, the Electric Propulsion Subsystem (EPS) comprises three core elements:

1. Thruster assembly which includes in this case the thrusters and the gimbals on which they can be mounted to control the thrust orientation. A specific mass of $2 \mathrm{~kg} / \mathrm{kW}$ together with a thrust to power ratio of 46 $\mathrm{mN} / \mathrm{kW}$ are considered in the calculations and the thrusters are sized with respect to the peak thrust delivered during mission.

2. Power Processing Unit (PPU) which supplies the high voltage current required for the ion engines to work efficiently. The Thruster Selection Unit (TSU) itself allows to select the thruster fed by the PPU. The $\mathrm{PPU} / \mathrm{TSU}$ is assumed to scale with the peak power during the mission with a specific mass amounting to $6 \mathrm{~kg} / \mathrm{kW}$.

3. Xenon Feed System (XFS) or Flow Control Unit (FCU) which usually includes a high pressure tank, a Xenon Control Assembly (XCA) which regulates the pressure and Xenon flow rate to the thrusters and the plenum tanks. The mass of the XFS is assumed to scale with the peak thrust with a specific mass of $1 \mathrm{~kg} / \mathrm{kW}$, which excludes the Xenon tank which itself is assumed to scale linearly with the propellant mass. The Dawn Xenon tank had a volume of 269L, could store up to $425 \mathrm{~kg}$ of Xenon and had a mass of $21.6 \mathrm{~kg}$, giving a tankage fraction of $5 \%$.

A parametric mass model was also considered for the other subsystems, including harness ( $5 \%$ of the wet mass), structure (20\% of the dry mass), AOCS ( $5 \%$ of the wet mass), as well as a non-scalable mass of $50 \mathrm{~kg}$ to telecommunications and data handling. Radiators are also scaled with respect to the maximum available power, considering a heat sink of $50 \%$ of the available power, ability of the radiator to re-radiate $400 \mathrm{~W}$ per square meter and an areal density of $5 \mathrm{~kg} / \mathrm{m}^{2}$. Eventually, any remaining mass is allocated to the additional propellant that will be used during the deflection phase (minus 


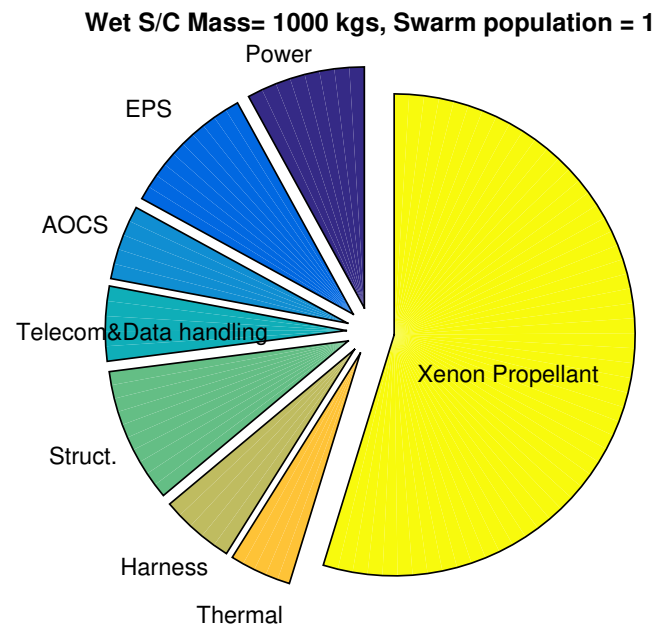

Figure 5: IBS mass budget for $T o F=847$ days and $t_{D}=-3615$ days before virtual impact with 2011AG5 and an oversizing factor of 1

the mass required to increase the size of the tanks and structure). If no mass is left prior to that step, the mission is considered infeasible with that particular combination of departure date, time of flight and oversising coefficient. Considering a wet mass of $1000 \mathrm{~kg}$, the transfer of Fig. 4, as well as an oversising factor of 1, Fig. 5 illustrates the resulting mass budget. The IBS spacecraft for this particular scenario would be able to generate a nominal thrust (in deflection mode) of $110 \mathrm{mN}$ and nominal input power level of 4.78 $\mathrm{kW}$ at a distance of 1 AU from the Sun.

The deflection phase starts as soon as the spacecraft has rendezvoused with the PHA. During that phase, it is assumed that the engines work at the maximum of their capability given the available power generated by the solar arrays at the current distance from the sun. Only half of the thrust can be used for the deflection as the other half is needed for station-keeping of the IBS. The acceleration on the asteroid is assumed to be also imparted in the tangential direction in average and is computed by

$$
u_{t}=\frac{F_{\mathrm{IBS}}}{m_{\mathrm{A}}}
$$

Knowing the thrust, the miss-distance can be computed following the procedure detailed in section 2.2. 
The time of the end of the application of the deflection action is set to the time of the virtual impact $t_{\text {MoID }}$ although if, at some point during the deflection, the propellant allocated to the deflection action goes to zero, the deflection action terminates and a null acceleration is considered for the remaining part of the integration.

\subsection{Laser Ablation}

The laser ablation deflection method aims at exploiting the material the asteroid is made of in order to generate the required thrust. The ablated material forms a plume of vaporized material which, due to the action/reaction principle, creates a controllable and continuous thrust on the asteroid.

With the power depending on efficient ( $30 \%$ from solar to electrical power) triple junction solar arrays, the level of thrust is again modulated by the square of the distance to the sun during the deflection phase, which is assumed to start as soon as the spacecraft arrives to the asteroid. The conversion from input power to ablative thrust $F_{a b l}$ on the asteroid is computed through the formula

$$
F_{L S}=\eta_{L S} C_{m} P_{i n}
$$

In which $\eta_{L S}$ is the electrical to optical (E/O) conversion efficiency of the laser system and $C_{m}$, the thrust coupling coefficient, which is known to vary between 10 to $100 \mu \mathrm{N} / \mathrm{W}_{\text {optical }}$ for most materials (Phipps, 2011). E/O efficiencies $>39 \%$ have already been demonstrated by multi-kW spectrally beam combined fiber-coupled diode lasers (Honea et al., 2013). Focused development under the DARPA SHEDs program has also lead to extremely high power conversion efficiency in the $9 \mathrm{xx}-\mathrm{nm}$ wavelength band, leading to diode bars with efficiency in excess of $74 \%$ and a clear route to efficiencies superior to $85 \%$ at room temperature (Crump et al., 2007). With demonstrated slope efficiency of optical fibers on the order of $80 \%$ (Jeong et al., 2004) and a demonstrated efficiency of spectral beam combining techniques of $91 \%$ (Drachenberg et al., 2011), we consider in this paper a global E/O efficiency of $50 \%$.

Detailed calculations performed on Forsterite by Thiry et al. (2016) indicate that the coupling coefficient of a CW laser operating under the plasma formation intensity near the 1 micron wavelength is dictated mainly by 2 parameters: the laser intensity $\Phi\left[\mathrm{W} / \mathrm{m}^{2}\right]$ which depends on the laser output power and focusing ability of the optics as well as the mean time available to heat the material which is roughly proportional to the ratio between the laser 


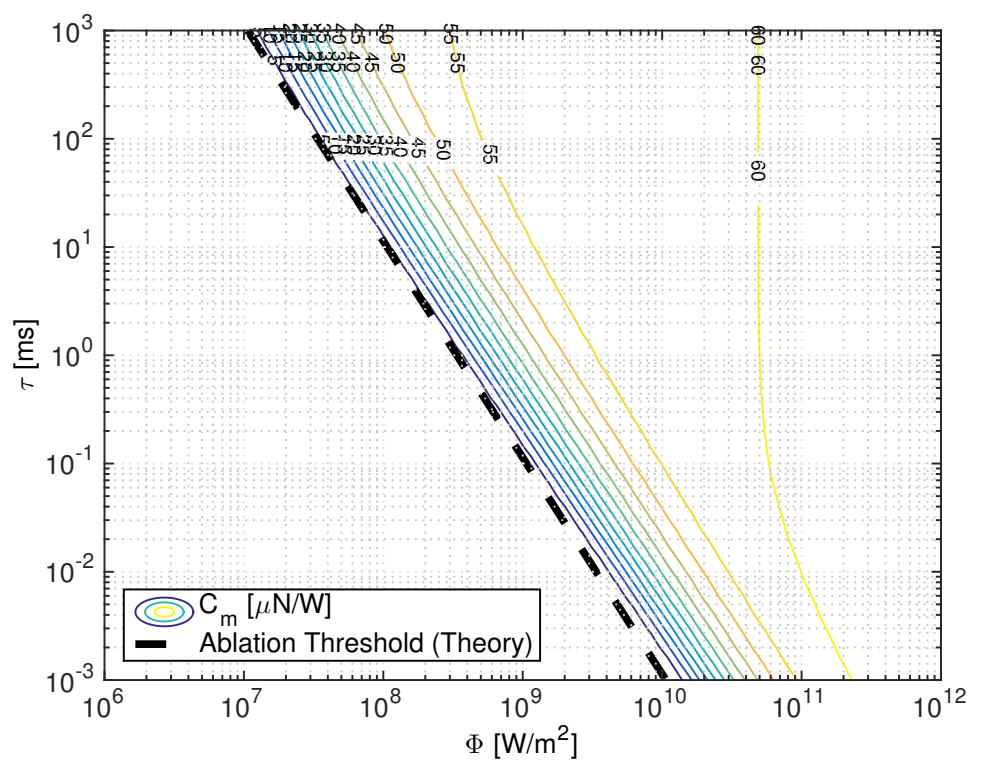

Figure 6: Thrust coupling coefficient $C_{m}$ as a function of the mean heating time $\tau$ and beam power density $\Phi$ for a CW laser from Thiry et al. (2016)

beam diameter and the relative speed of the asteroid surface with respect to the laser beam (on the order of $6 \mathrm{~cm} / \mathrm{s}$ if one considers the spin-limit of a $156 \mathrm{~m}$ asteroid). Fig. 6 shows the result of these calculation for Forsterite, a main constituent of S-type asteroids which are thought to dominate the population in the inner belt. For typical mean heating times on the order of 10-100 ms and typical CW laser beam intensities on the order of $1 \mathrm{GW} / \mathrm{m}^{2}$ envisioned in our laser system, one can see from this chart that the thrust coupling has a value around $55-60 \mu \mathrm{N} / \mathrm{W}$ and will only be weakly affected by the temporal changes in operating conditions due to the variation of input power with respect to the square distance to the sun. To generate the intensity levels required, the optics should be designed using the diffraction limit focusing capability at the shooting distance. The optical components should also be designed so that they are exposed to intensity levels well under their damage threshold. As an example, an optics with a primary mirror of $60 \mathrm{~cm}$ diameter would be enough to generate a $3 \mathrm{~mm}$ laser spot at a $1 \mathrm{~km}$ shooting distance. For a $10 \mathrm{~kW}$ laser, this would correspond to an intensity of 1.4 $\mathrm{GW} / \mathrm{m}^{2}$ at the focal spot, but only $35 \mathrm{~kW} / \mathrm{m}^{2}$ on the primary mirror.

Plasma effects are also not expected to play any role under CW laser irra- 


\begin{tabular}{rlllllll}
\hline Symbol: & $\mathrm{Mg}$ & $\mathrm{Fe}$ & $\mathrm{Si}$ & $\mathrm{O}$ & $\mathrm{C}$ & $\mathrm{Mg} 2 \mathrm{SiO} 4$ & $\mathrm{Fe} 2 \mathrm{SiO} 4$ \\
\hline Name: & Magnesium & Iron & Silicon & Oxygen & Carbon & Forsterite & Fayalite \\
$\mathrm{A}:$ & 24 & 26 & 28 & 16 & 12 & - & - \\
$C_{m p}^{\text {opt }}(\mu \mathrm{N} / \mathrm{W}):$ & 80 & 83 & 86 & 67 & 59 & 75 (avg.) & 79 (avg.) \\
\hline
\end{tabular}

Table 1: Optimal thrust coupling predicted by Eq.13 for various materials encountered in asteroids

diation below intensity levels of $10 \mathrm{GW} / \mathrm{m}^{2}$, which are required to accelerate the free electrons in the vapor by inverse Bremsstrahlung until their kinetic energy becomes sufficient to ionize the atoms of the vapor by an avalanche process, according to Poueyo-Verwaerde et al. (1993). A model to predict the thrust coupling coefficient in the Plasma regime has been developed by Phipps et al. (1988) for pulsed laser systems and it is interesting to compare the peak coupling predicted by this model with the values predicted by our $\mathrm{CW}$ model. In this model, the plasma coupling $C_{m p}$ coefficient was empirically found to follow a power law:

$$
C_{m p}(\mu N / W) \approx 184 \frac{\Psi^{9 / 16}}{A^{1 / 8}(\Phi \lambda \sqrt{\tau})^{1 / 4}}
$$

in which $\tau$ is the pulse duration, $\lambda$ the laser wavelength and $\Psi$ depends on the average atomic number $\mathrm{A}$ and the average ionization state $\mathrm{Z}$ as:

$$
\Psi=\frac{A}{2\left(Z^{2}(Z+1)\right)^{1 / 3}}
$$

Phipps et al. (1988) noted that the optimal coupling happens for values of intensity and pulse duration such that $\Phi \sqrt{\tau}=8.5 \mathrm{E}+08 \mathrm{Ws}^{1 / 2} / \mathrm{m}^{2}$, which is about twice the value at which plasma ignition occurs for pulses $<1 \mathrm{~ms}$. Initially, ionization enhances the thrust coupling coefficient because, despite requiring more energy to ionize the plume, increased absorption by the plume increases the thermal coupling coefficient (Rosen et al., 1982). Using the same estimation as Phipps (2014), we assumed a single stage ionization $(\mathrm{Z}=1)$ and injected the relevant values in Eq. (13) to compute the optimal thrust coupling coefficient for a wide range of atoms and materials encountered in asteroid for a $1 \mu \mathrm{m}$ laser wavelength. Our results are summarized in Table 1. Interestingly, the value predicted is about $25 \%$ higher than our own calculation, which neglected plasma effects. In the rest of this paper, we 
will consider a conservative value of $40 \mu \mathrm{N} / \mathrm{W}$, accounting also for possible loss due to the shape irregularity of the asteroid and the fact that the laser ablation thrust can only be oriented in the desired direction tangential with respect to the PHA trajectory in average. In fact, as explained by Vetrisano et al. (2015, 2016) a smart laser steering strategy would allow to improve further the thrust directional efficiency.

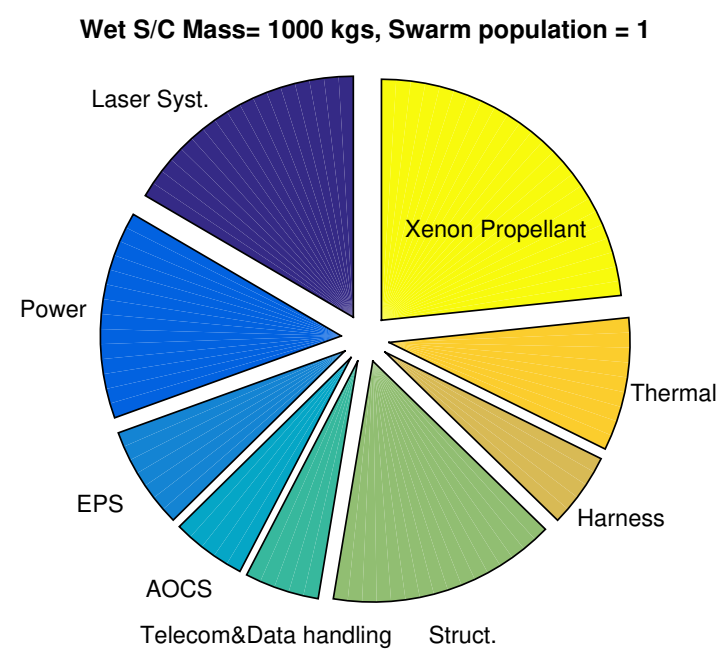

Figure 7: LS mass budget for $T o F=847$ days and $t_{D}=-3615$ days before virtual impact with 2011AG5

As in the case of the IBS method, a low-thrust transfer is assumed for this method, which allows using the large power available (the laser is only used once the spacecraft has rendezvoused with the asteroid) to be used by an electric propulsion system during the transfer phase. Initially, the preliminary sizing of the different subsystems is parametrized in the same way as with the IBS method (without oversizing coefficient). Some of the remaining dry mass available is then allocated to the laser system in order to match with the available power or, if no mass was left at this point, this particular mission is considered non-feasible. It is assumed that the laser system (including optics) scales with the peak power with a specific mass of $15 \mathrm{~kg} / \mathrm{kW}$ (current fibre-coupled diode laser are already available with a specific mass of $1 \mathrm{~kg} / \mathrm{kW}$ for welding applications). If there is still mass left after this step, the size of the power system, laser system and radiators is increased until all the mass has been allocated. Considering the same scenario 
as for the IBS, a summary of the mass budget considering a wet mass of 1000 $\mathrm{kg}$ is given in Figure 7. For this specific case, the laser system would deliver an estimated nominal ablative thrust of $168 \mathrm{mN}$ and nominal input power level of $8.4 \mathrm{~kW}$ at a distance of $1 \mathrm{AU}$ from the Sun. For comparison, NASA's Dawn spacecraft, which recently visited the dwarf planet Ceres using 3 NSTAR gridded ion-thrusters and achieved a record cumulated $\Delta v$ of $14 \mathrm{~km} / \mathrm{s}$, had a wet mass of $1240 \mathrm{~kg}$ with $425 \mathrm{~kg}$ of Xenon propellant, a dry spacecraft mass of $815 \mathrm{~kg}$ and a solar array of $36.4 \mathrm{~m}^{2}$ able to deliver $10.3 \mathrm{~kW}$ at $1 \mathrm{AU}$.

Finally, the deflection is computed as in the case of the IBS, except that the acceleration is imparted for the whole duration until the virtual impact epoch $t_{\text {MOID }}$. As in the case of the IBS method, tugging forces are considered negligible at the operation distance (less than $3 \mathrm{mN}$ for distances larger than $1 \mathrm{~km})$.

\subsubsection{Contamination Considerations}

According to previous studies (Gibbings et al., 2013), the impingement with the plume of gas and debris, generated by the ablation process, could build up enough material on the surface of the solar arrays to reduce the output power below the ablation threshold. At the same time it was shown that this contamination has a limited impact on the laser itself and related optics. Furthermore, as shown in the ESA LightTouch2 study (Vasile et al., 2013), by properly positioning the spacecraft with respect to the asteroid, aligning the arrays with the plume and adding Whipple shields the effect of contamination can be mitigated to the point that they can be considered negligible over the lifetime considered in this paper.

\subsection{Gravity Tractor}

The Gravity Tractor (GT) exploits the mutual attraction between the spacecraft and the asteroid to progressively change the velocity of the asteroid. In the more traditional configuration, the spacecraft is placed at a distance $d$ from the asteroid and two thrusters mounted in a slanted configuration, to avoid thrust impingement, would balance the gravity attraction. By doing so the net result is a constant acceleration on a compound spacecraft-asteroid, see Figure 8. For the position of the spacecraft to be 
fixed, the gravity force must equate the net thrust $F_{\text {hover }}$ :

$$
\begin{aligned}
F_{\text {hover }} & =2 T_{S} \cos \left(\arcsin \left(\frac{R_{a}}{d}\right)+\phi\right) \\
F_{g} & =\frac{G m_{a} m_{S}(t)}{d^{2}} \\
F_{\text {hover }} & =F_{g}
\end{aligned}
$$

where $T_{S}$ is the thrust of a single engine in the slanted configuration, $\phi$ the half-divergence angle of the engine, $R_{a}$ is the radius of the asteroid, $m_{a}$ its mass, $G$ the gravity constant and $\left.m_{(} t\right)$ is the mass of the spacecraft at time $t$. The tugging acceleration is simply:

$$
u_{g t u g}(t)=\frac{G m_{S}(t)}{d^{2}}
$$

If the engines are assumed to be always on and the initial mass of the spacecraft is $m_{i}$, the mass of the spacecraft at time $t$ can be expressed as:

$$
m_{S}(t)=m_{i} \exp \left(-\frac{G m_{a}\left(t-t_{0}\right)}{d^{2} \cos \left(\arcsin \left(\frac{R_{a}}{d}\right)+\phi\right) I_{s p} g_{0}}\right)
$$

where $I_{s p}$ is the specific impulse of the engine and $g_{0}$ the gravity acceleration on the surface of the Earth and $t_{0}$ is the beginning of the deflection action.

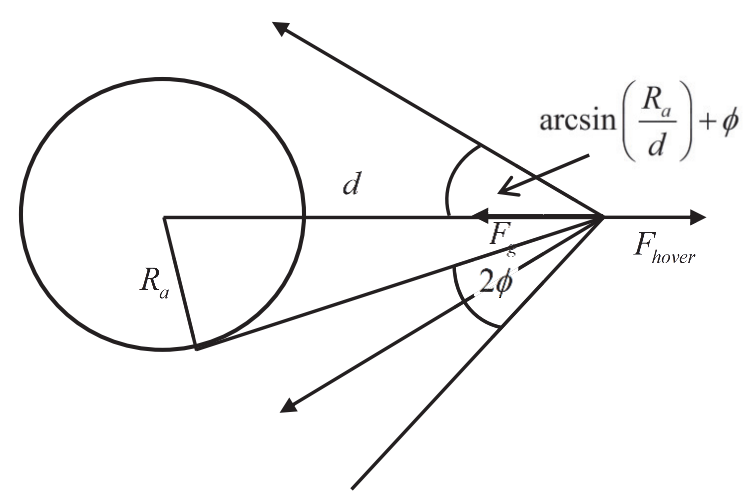

Figure 8: Sketch of the gravity tug approach with slanted engines 


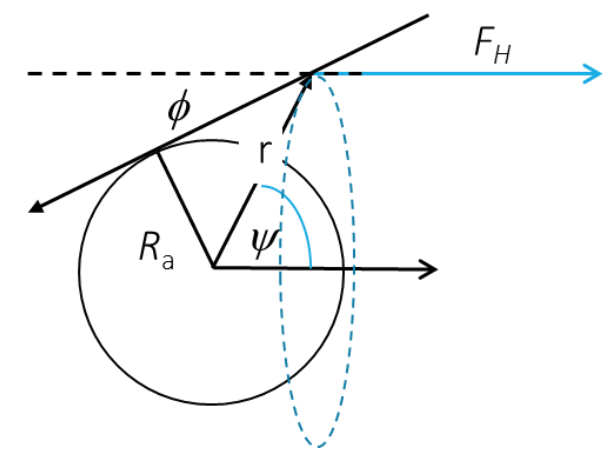

Figure 9: Sketch of the gravity tug approach with halo configuration

In McInnes (2007) and Yamaguchi and Yamakawa (2014) a variant of the original gravity tractor concept was proposed to remove the need for a slanted configuration and to use the thrust more efficiently. The idea is to place the tractor on a displaced halo orbit that is artificially maintained by a constant thrust. If the plume of gas of the engine generating the thrust is not impinging the asteroid, the net result is traction on the asteroid in the direction of the thrust vector (see Figure 9). Compared to the slanted configuration, the halo configuration requires only one engine but the thrust delivered by the engine has to be lower or the distance from the asteroid has to be shorter.

With reference to Figure 9, the achievable tugging effect as a function of the divergence angle $\phi$ is:

$$
u_{H}=\frac{G m_{H}(t)}{R_{a}^{2}} \cos \psi \sin (\psi-\phi)^{2}=\frac{G m_{H}(t)}{R_{a}^{2}} \tau
$$

The $\tau$ factor in (20) is represented in Figure 10 for different values of $\phi$.

The figures shows that $\tau$ cannot be 1 for any value of $\psi$. The maximum is in fact about 0.385 and is realised when $\phi=0$. In comparison the slanted configuration can reach a theoretical $\tau=1$ for an infinite thrust.

In order to get an estimation of the mass of propellant required for the halo configuration in comparison to the slanted configuration, one can consider the simple case in which the distance of the slanted configuration from the centre of the asteroid is kept constant. In this case, the mass of the 


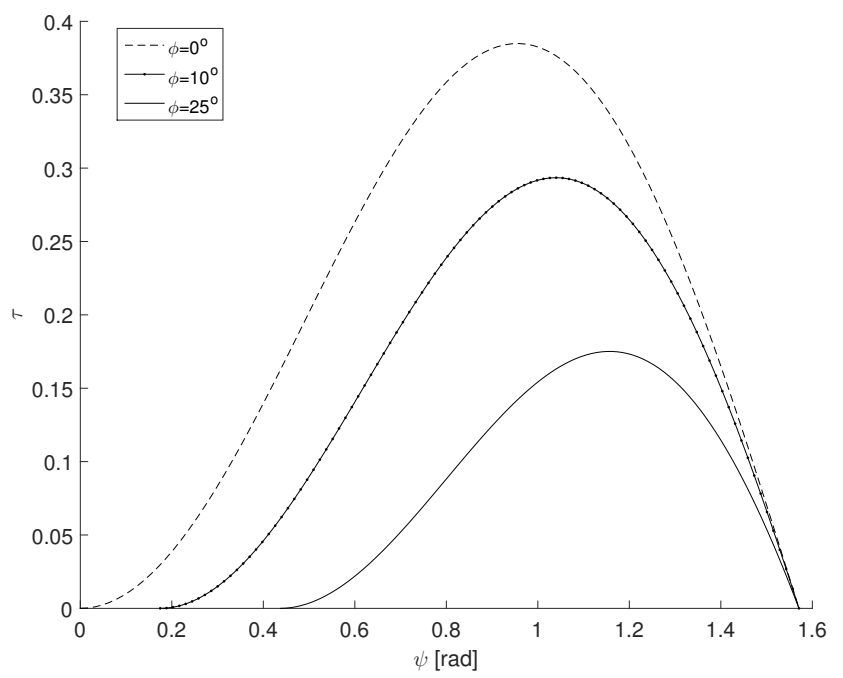

Figure 10: $\tau$ factor as a function of $\psi$

spacecraft is given by Eq. (19) and the tugging force is:

$$
F_{g}=\frac{G m_{a} m_{i} e^{-\frac{G m_{a} \Delta t}{I_{s p} g_{0} d^{2} \cos \alpha}}}{d^{2}}=2 T_{S} \cos \alpha=T_{H}
$$

where $\alpha=\arcsin \left(\frac{R_{a}}{d}\right)+\phi, \Delta t=t-t_{0}$ and the further assumption is that the thrust of the halo configuration follows the same time law of the tugging force given by the slated configuration. As a result the variation of mass of the halo configuration is:

$$
\dot{m}_{H}=-c \frac{G m_{a} m_{i} e^{\frac{-c G m_{a} \Delta t}{d^{2} \cos \alpha}}}{d^{2}}=-\frac{c G m_{a} m_{i}}{d^{2}} e^{-A \Delta t}
$$

with $c=1 /\left(I_{s p} g_{0}\right)$ and:

$$
A=\frac{c G m_{a}}{d^{2} \cos \alpha}
$$

The mass of the halo spacecraft as a function of time results to be:

$$
m_{H}-m_{H 0}=\frac{c G m_{a} m_{i}}{d^{2}} \frac{e^{-A \Delta t}}{A}=m_{i} \cos \alpha e^{-A \Delta t}-m_{i} \cos \alpha
$$

If the initial mass of the halo and slanted configurations are the same, then:

$$
m_{H}=m_{S} \cos \alpha
$$




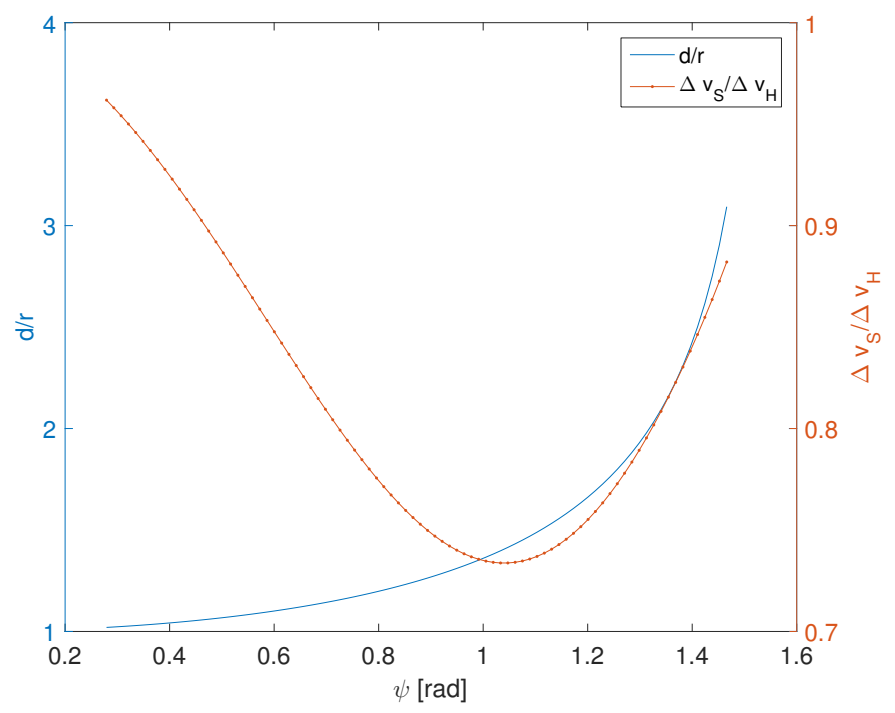

Figure 11: Relative distance and $\Delta v$ for two GT configurations

Given that both configurations generate the same tugging force, the total time to achieve a given deflection is the same and, therefore, the mass of the halo configuration is simply $\cos \alpha$ lower than the mass of the slanted configuration.

The question is now whether for a fixed distance and equal traction the halo configuration can deliver a higher or lower $\Delta v$ than the slated configuration. To this end, one can calculate the distance $d$ that provides the same traction $a_{H}$ for different $\psi$ and the corresponding $\Delta v$ for the two configurations. Given equation (20) one can calculate the mass of the halo spacecraft as:

$$
m_{H}(t)=m_{H 0} e^{\frac{G m_{a} \tau}{R_{a}^{2} I_{s p} g_{0}} t}
$$

From the mass consumption one can derive the time at which the deflection action stops by assuming a mass fraction for the available propellent:

$$
t_{H}=\log \left(\frac{m_{H}\left(t_{H}\right)}{m_{H 0}}\right) \frac{R_{a}^{2} I_{s p} g_{0}}{G m_{a} \tau}
$$

and by replacing the mass $m_{H}$ in (20) and integrating in time till $t_{H}$ one gets 
the $\Delta v_{H}$, which is simply:

$$
\Delta v_{H}=\frac{m_{H 0} e^{\frac{G m_{a} \tau}{R_{a}^{2} I_{s p} g_{0}} t_{H}}}{m_{a}}=\frac{m_{H}\left(t_{H}\right)}{m_{a}}
$$

A similar procedure can be used to calculate the $\Delta v_{S}$ inserting (19) into (18) and integrating with respect to time. Figure 11 shows the ratio between the $\Delta v_{S}$ delivered by the slant configuration and the one delivered by the halo configuration. On the same plot the ratio between the distance $d$ and the distance $r$ is also shown. As $\psi$ goes to $\pi / 2, r$ approaches $R_{a}$ but the traction goes to zero therefore $d$ goes to infinity. On the other hand as $\psi$ approaches 0 asymptotically both $r$ and $d$ go to infinity. The figures shows that for a fixed distance and equal traction the halo configuration always delivers a higher $\Delta v$. Note however that the traction time is proportionally longer for the halo configuration. For a reference $\Delta v$ of $1 \mathrm{~km} / \mathrm{s}$ the slant configuration is 6 to $7 \%$ faster than the halo configuration.

In conclusion while the halo configuration uses less propellant mass for the same traction, the slanted configuration can achieve higher tractions in a shorter amount of time.

In the following we will use the halo configuration with an assumed divergence angle of $10^{\circ}$ for our comparison as in Sanchez et al. (2009) an optimised slanted configuration was considered instead. Practically speaking, our model of the GT method was adapted from the IBS model to consider a time-dependent maximum tugging thrust during the integration of the deflected orbit. This model also considers that engines are only required on one side of the GT (for station keeping) contrary to the IBS which requires engines on both sides of the spacecraft.

\section{Theoretical Considerations}

In this section we provide a theoretical analysis of the energy required to increase the transfer of momentum during impact. In other words we consider the case in which the orbit of the impactor is modified so that the relative velocity between spacecraft and asteroid at impact is increased.

Consider a simple planar case in which the expected impact of the asteroid with the Earth is at an apsidal point. The impactor spacecraft is ramming

into the asteroid at the same apsidal point with $\beta=1$. The variation of 
velocity of the asteroid post impact with the spacecraft is:

$$
\delta v=\frac{m_{s / c}}{m_{a}}\left(\|q\|-\Delta v_{T}\right)
$$

where $q$ is the difference in velocity due to the difference between the orbit of the asteroid and the one of the Earth:

$$
q=\sqrt{\frac{2 \mu}{r_{E}}-\frac{\mu}{a_{a}}}-\sqrt{\frac{\mu}{r_{E}}}
$$

and $\Delta v_{T}$ is the increment due to the transfer of the spacecraft on a suitable orbit:

$$
\Delta v_{T}=\sqrt{\frac{2 \mu}{r_{E}}-\frac{\mu}{r_{E}+\delta a_{N}}}-\sqrt{\frac{\mu}{r_{E}}}
$$

The mass of the spacecraft at impact $m_{s / c}$ can be related to the mass at launch $m_{s i}$ through:

$$
\frac{m_{s / c}}{m_{s i}}=e^{-\frac{\Delta v_{T}}{I_{s p} g_{0}}}
$$

from which:

$$
\delta v=\frac{m_{s i} e^{-\frac{\Delta v_{T}}{I_{s p} g_{0}}}}{m_{a}}\left(\|q\|-\Delta v_{T}\right)
$$

Figures $12 \mathrm{a}$ and $12 \mathrm{~b}$ show the $\delta v$ for different asteroid semi-major axes, $a_{a}$, and different $\Delta v_{T}$, in the case of an $I_{s p}=300 \mathrm{~s}$ and an $I_{s p}=3000 \mathrm{~s}$ respectively. The figures show that for a low $I_{s p}$ the optimal strategy is not to perform any transfer as the increase in momentum is proportional to $\Delta v_{T}$ but the loss in mass is proportion to $e^{-\Delta v_{T}}$. This is a problem for asteroids with semi-major axis close to $1 \mathrm{AU}$, low eccentricity and low inclination as a transfer produces a low gain in $\delta v$ and a further increase in $\Delta v_{T}$ might in fact lead to a decrease in the deflection.

The situation appears to be different for a high $I_{s p}$. In this case the gain provided by the transfer for high $a_{a}$ is limited but the one provided for low $a_{a}$ becomes interesting. Note that this analysis does not consider gravity losses and the time required to deliver the required $\Delta v_{T}$. Figure 13 shows the difference between the $\Delta v$ required to rendezvous with the asteroid and $\Delta v_{T}$. A positive $\Delta \Delta v$ indicates that a rendezvous is more expensive. Negative areas, instead, suggest that a rendezvous is a potential option. The associated $-\Delta \Delta v$ is then available to achieve a deflection with, for example, a 
slow push method. The figure gives an indication of when a kinetic impactor might be preferable to a slow-push technique. Another qualitative indication can be obtained by computing the required propellant mass to generate the same change in linear momentum given by a kinetic impactor. Assuming a constant low-thrust push on an asteroid, the mass of propellant required to deliver the variation of linear momentum in Eq. (32) is given by:

$$
\Delta m I_{s p} g_{0}=m_{s i} e^{-\frac{\Delta v_{T}}{I_{s p} g_{0}}}\left(\|q\|-\Delta v_{T}\right)
$$

to this, one has to add the mass required to inject the spacecraft into the orbit of the asteroid:

$$
\Delta m_{L T}=m_{s i} \frac{e^{-\frac{\Delta v_{T}}{I_{s p} g_{0}}}\left(\|q\|-\Delta v_{T}\right)}{I_{s p} g_{0}}+m_{s i}\left(1-e^{-\frac{q}{I_{s p} g_{0}}}\right)
$$

The mass of the propellent on the kinetic impactor is instead given by Eq. (32) which then gives the relative mass fraction:

$$
\frac{\Delta m_{L T}}{\Delta m_{K I}}=\frac{\frac{e^{-\frac{\Delta v_{T}}{I_{s p} g_{0}}}\left(\|q\|-\Delta v_{T}\right)}{I_{s p} g_{0}}+\left(1-e^{-\frac{q}{I_{s p} g_{0}}}\right)}{\left(1-e^{-\frac{\Delta v_{T}}{I_{s p} g_{0}}}\right)}
$$

The relative mass fraction for an $I_{s p}=3000$ s can be seen in Figure 14 . The figure shows that, in this particular case, when the orbit of the asteroid approaches the one of the Earth the slow-push solution is up to $20 \%$ more efficient, which translates into $20 \%$ more deflection action, than increasing the energy of the kinetic impactor, albeit with a low-thrust propulsion system. It has to be noted that although this analysis is limited to a special case, some considerations are generally applicable. In particular, a highly inclined orbit favours a kinetic impactor in the same way an orbit with a high $a_{a}$ does. On the contrary, for a shallow crosser with a low inclination, the mass loss coming from the rendezvous with the asteroid is limited compared to the increase in orbital energy of the kinetic impactor. Finally, if the slow-push action did not require any propellent at all the first term on the right hand side of Eq. (35) would translate in an additional \% of deflection.

The analysis in this section is only qualitative but suggests that the lowthrust impactor proposed by some authors in the past (Conway (1997)) can be a valid alternative for some classes of asteroids, while for others, a simple direct injection with a single impulse is potentially optimal. The use of a 
low-thrust transfer for the kinetic impact become progressively more interesting as the propellant cost decreases (or the $I_{s p}$ increases). For this reason, solutions using solar sails or electromagnetic sails have been considered in the past. These solutions, however, require careful considerations on the design of the navigation and control system to guarantee a successful impact at hyper-velocity due to the limited control authority. At the same time one can argue that if a slow push method requires low or zero propellant to deliver the required deflection action, then that method might be optimal in the case of low semimajor axis, low eccentricity and low inclination asteroids. Furthermore, if one combines a low-cost transfer with a zero-propellent slow push method then slow push become optimal for a wide range of targets.

In the following we will limit our comparison only to the simpler version of the kinetic impactor leaving the comparison with the low-thrust counterpart to a future work. 


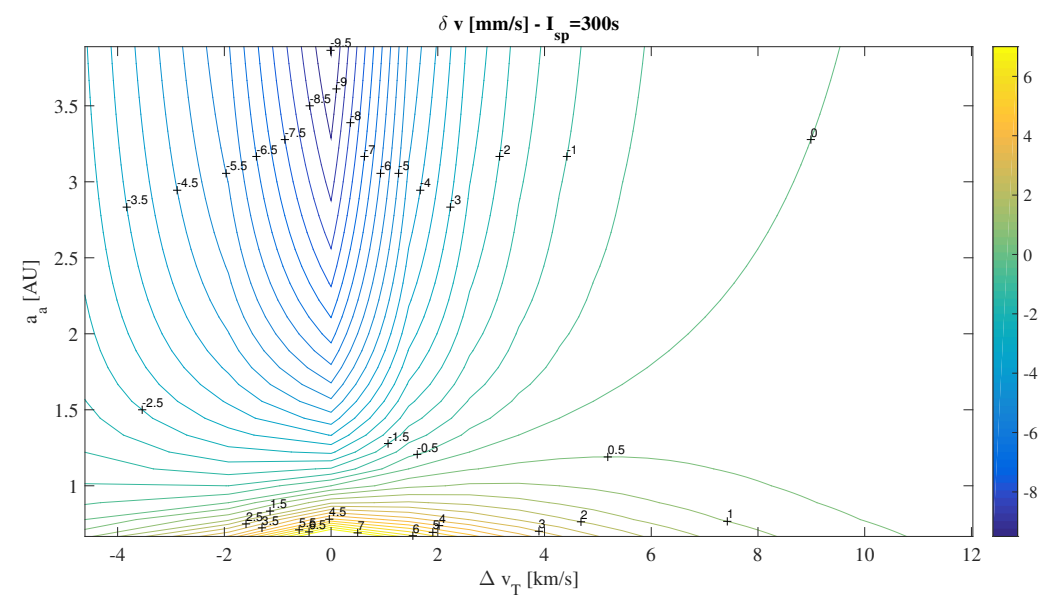

(a) $I_{s p}=300 \mathrm{~s}$

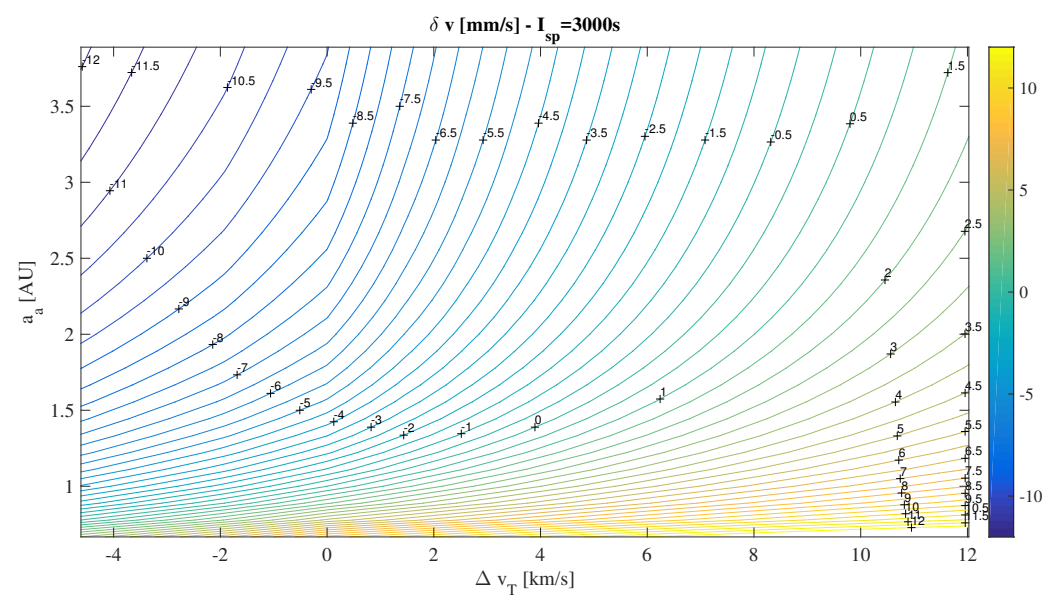

(b) $I_{s p}=3000 \mathrm{~s}$

Figure 12: Deflection $\delta v$ for different semi-major axes and different $\Delta v_{T}$ : a) contour lines of achievable $\delta v$ imparted onto the asteroid given a departure $\Delta v_{T}$ and asteroid semimajor axis $a_{a}$ for an engine $I_{s p}=300 \mathrm{~s}, \mathrm{~b}$ )contour lines of achievable $\delta v$ imparted onto the asteroid given a departure $\Delta v_{T}$ and asteroid semi-major axis $a_{a}$ for an engine $I_{s p}=3000 \mathrm{~s}$ 


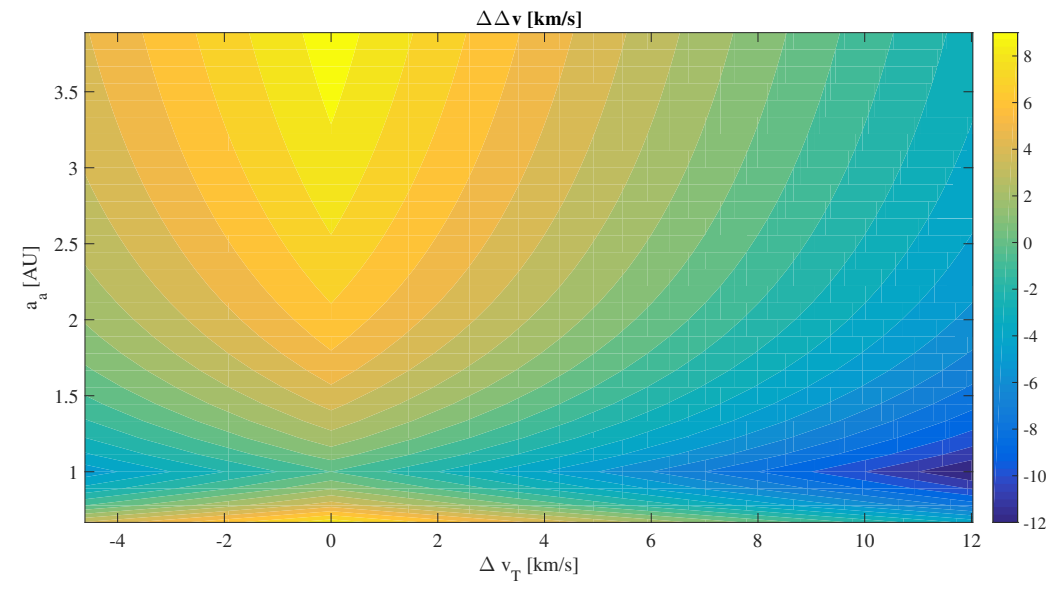

Figure 13: $\Delta \Delta v$ for different semi-major axes and different $\Delta v_{T}$ : negative values indicate that a low-thrust tug is preferable to a kinetic impactor.

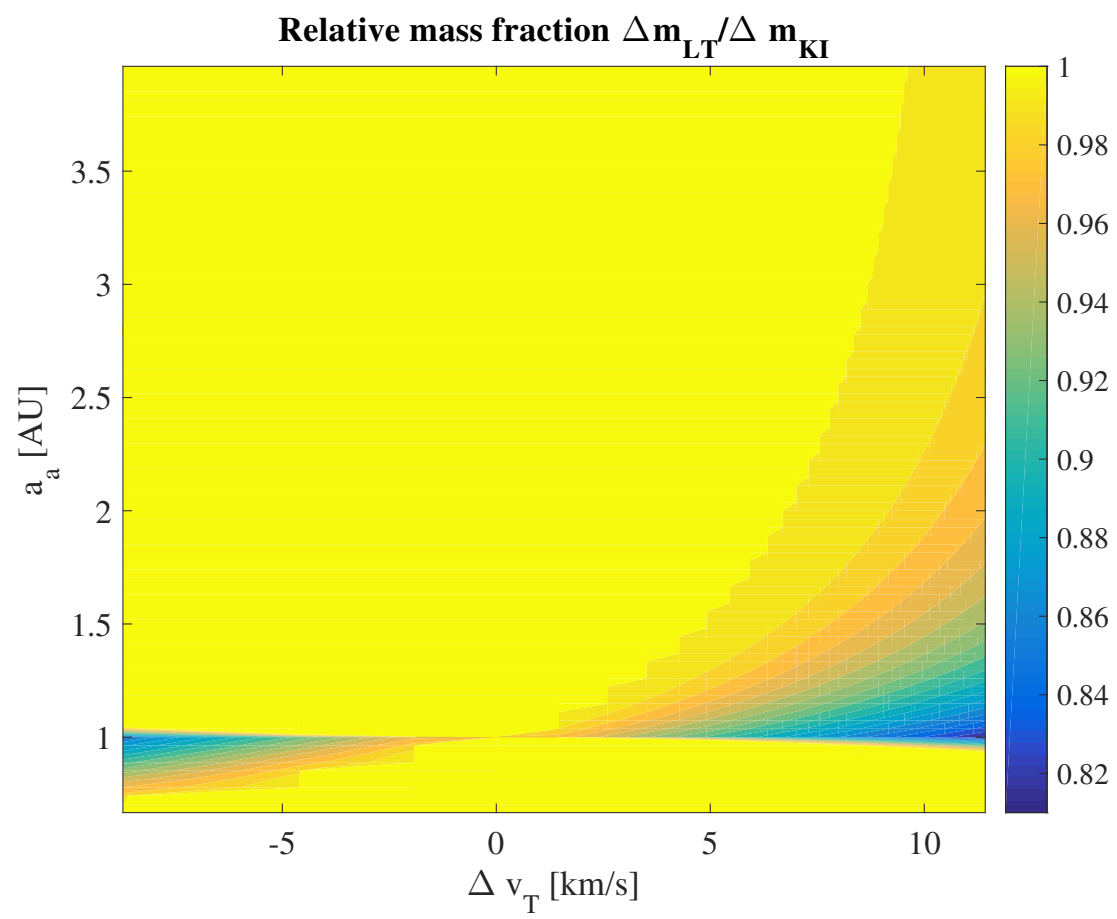

Figure 14: Relative mass fraction between low-thrust tug and kinetic impactor: fraction between the mass of propellant required to deflect and asteroid with low-thrust tug and the mass of propellant required to deflect by the same amount the same asteroid but with a kinetic impactor. 


\section{Asteroid Sampling Strategy}

This section explains how we selected the asteroids forming the test set to compare the performance of different deflection methods.

\subsection{PHA Distribution}

As in the work of Bach (2012), the undeflected motion of the PHAs considered in this work is approximated by Keplerian orbits in a heliocentric frame and the Earth orbit is approximated with an exact circle of radius 1AU. Intuitively, this simplification induces two necessary but not sufficient conditions on the semi-major axis $a$ and eccentricity $e$ for impacting PHAs:

$$
a(1-e)<1 \mathrm{AU} \text { and } a(1+e)>1 \mathrm{AU}
$$

Using the criterion in Eq. (37), we extracted 8273 Earth-crossing NEAs from the NEODyS database presently maintained at the University of Pisa ${ }^{2}$. The distribution of these NEAs can be seen on Fig. 15 where the green lines represent the necessary crossing condition of Eq. (37).

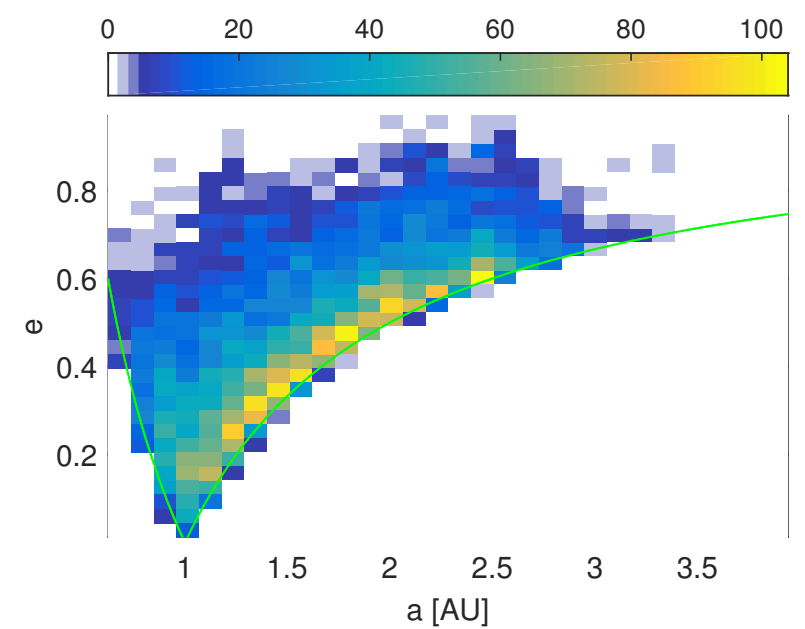

Figure 15: Distribution in semi-major axis and eccentricity of all known NEAs with an orbit crossing the heliocentric sphere of radius $1 \mathrm{AU}$

\footnotetext{
${ }^{2}$ http://newton.dm.unipi.it/neodys
} 


\subsection{Virtual Impactor Model}

Fixing the semi-major axis, eccentricity and inclination with their actual value from the extracted database, one independent element remaining to fix is the longitude of the ascending node node $\Omega$ of the PHA's orbital plane with respect to the ecliptic. However, since we neglect the small minute Earth orbit eccentricity, the impact epoch is arbitrary and we can choose to fix $\Omega=0$ so that the PHA's orbital planes crosses the ecliptic along the vernal equinox direction. The last parameters to fix are the argument of perihelion $\omega$ and the true anomaly $\theta$ of the PHA at the impact epoch $t_{\text {MoID }}$. From the above simplifications, the argument of perihelion and the true anomaly may only adopt two distinct values to respect the impact condition:

$$
1 \mathrm{AU}=\frac{a\left(1-e^{2}\right)}{1+e \cos \omega} \text { and } \theta=2 \pi-\omega
$$

The two solutions of Eq. (38) correspond to an impact with the ascending or the descending branch of the PHA respectively.

\subsection{Sampling Strategy}

We formed a sample of virtual impactors by randomly selecting 100 PHAs in the NEODyS database, using the method described in Sec. 5.2 and considering an equal probability of impact with the ascending or the descending branch of the PHAs. The distributions in semi-major axis, eccentricity and inclination of this test sample are plotted for further reference in Fig. 16.

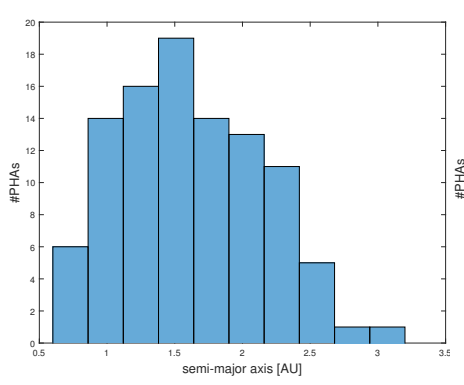

(a) semi-major axis

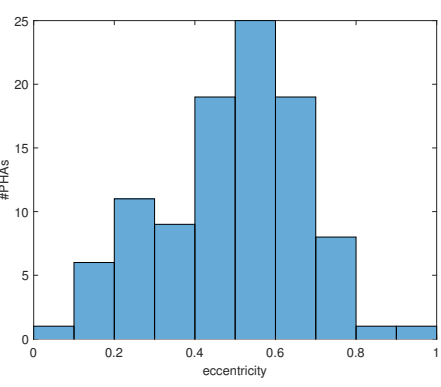

(b) eccentricity

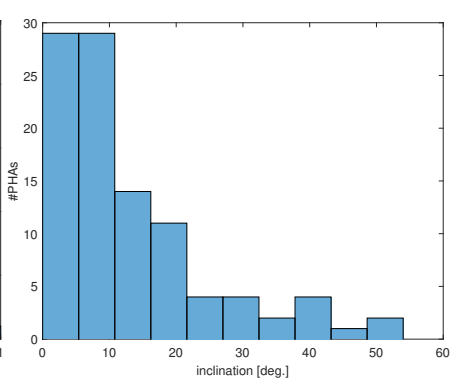

(c) inclination

Figure 16: Distribution of the 100 PHAs randomly sampled from the NEODyS database 


\section{Global Optimisation Strategies}

The optimisation of the deflection strategies requires the global exploration of the parameter space. Furthermore, it is desirable to investigate the trade-off between warning time and achievable miss distance. For this reason we used two global optimisation procedures one for single objective and the other for multi-objective optimisation of multi-modal functions: MP-AIDEA (Di Carlo et al., 2015) and MACS2 (Ricciardi and Vasile, 2015). In the following we briefly present how each optimisation approach works and how it was used in the context of this paper.

\subsection{Optimisation with MP-AIDEA}

For all methods, the impact parameter can be computed as a strongly non-linear function of the departure date $t_{D}$ and the time of flight $T_{o} F$, but also the oversizing coefficient in the case of the IBS method. For each mission scenario we globally explore the space of possible departure dates, transfer times and oversizing coefficients (in the case of the IBS) with a memetic algorithm called multi-population adaptive inflationary differential evolution algorithm (MP-AIDEA).

MP-AIDEA is a multi-population adaptive version of Inflationary Differential Evolution. Inflationary Differential Evolution is based on a simple but theoretically rigorous restart rule that allows an effective evolutionary heuristic, like Differential Evolution (DE), to avoid stagnation. In MP-AIDEA a number of populations, each one composed by a number of virtual agents, evolve in parallel in search of the global optimum. At each step of evolution, each agent uses DE heuristics to move from one site to another of the solution space and evaluates a new potential solution (a new possible combination of decision variables). The basic idea is to restart the evolutionary process when the populations contract within a given area in the solution space. At every restart of the evolutionary process a local search is run from the best individual in each population. All the discovered local minima are then stored in an archive. The restart process is such that the populations are initialised outside a trust region enclosing clusters of the already discovered minima in the archive. MP-AIDEA extends this concept by automatically adapting some key parameters governing the convergence of the algorithm. MP-AIDEA has been extensively tested on a range of difficult problems including real-world applications (Di Carlo et al., 2015). 
The decision variables handled by MP-AIDEA are the time of flight, ToF, and mission duration, $t_{\text {mission }}=t_{\mathrm{MOID}}-t_{D}$, and are limited by box constrains. The time of flight represents the time between mission departure from the Earth and arrival at the satellite while the mission time is the total time between Earth departure and the time of virtual impact between the asteroid and the Earth. Table 2 reports for each optimisation associated to each deflection method (KI=Kinetic Impactor, IBD=Ion Beam Shepherd, $\mathrm{LA}=$ Laser Ablation), the box constraints on the decision variables ToF and $t_{\text {mission }}$, the number of agents per population used by MP-AIDEA to search for the global optimum (\#agents), the number populations (\#pop) and total number of calls to the objective function (\#fevals).

\begin{tabular}{rlllll}
\hline Method & ToF (days) & $t_{\text {mission }}$ (days) & \#agents & \#pop & \#fevals \\
\hline KI & {$[0,1000]$} & {$[1000,3662.42]$} & 10 & 4 & 10000 \\
IBS & {$[300,2000]$} & {$[2000,3662.42]$} & 15 & 4 & 1200 \\
LA & {$[300,2000]$} & {$[2000,3662.42]$} & 10 & 4 & 1000 \\
\hline
\end{tabular}

Table 2: Parameters and box constrains used during the optimisation with MP-AIDEA

During each function evaluation, we also run an internal loop to evaluate the solution within the feasible range of number of revolutions for the trajectory computed by the Lambert solver and the spherical shaping algorithm. When more than one transfer are feasible for the combination of $T_{o} F$ and $t_{D}$, our fitness function only returns the solution with the number of revolution that provides the best miss-distance.

\subsection{Optimisation with MACS2}

Multi-Agent Collaborative Search (MACS2) is a memetic multi-objective optimisation framework that aims at identifying the set of Pareto optimal solutions. A solution $x$ is said to be (weakly) Pareto optimal if there is no other solution $y$ whose associated objectives are all better than the ones associated to $x$. The set of Pareto optimal solutions is called Pareto set and the set of values of the objective functions associated to Pareto optimal solutions forms the Pareto set. In MACS2 a population of virtual agents implements a number of local search heuristics intermingled by global communication heuristics that help the population to reconstruct an approximation of the Pareto set. Each agent explores a neighbourhood of the parameter (or solution) space, 
stores Pareto optimal solutions in an archive and shares information with the other agents in the population. For more specific information please refer to Ricciardi and Vasile (2015).

MACS2 was used to optimise both the mission duration and the impact parameter. Therefore, one objective function was defined as:

$$
o b j_{1}=\left\{\begin{array}{l}
\left(b-2 R_{e}\right)^{2} \text { if } b<2 R_{e} \\
0 \text { otherwise }
\end{array}\right.
$$

This definition forces the optimiser to find minimum time solutions that achieve at least a 2 Earth radii deflection. The other objective function was defined as:

$$
o b j_{2}=\mathrm{ToF}+t_{d e f l}
$$

where $t_{d e f l}=t_{\text {MOID }}-t_{d}$. Table 3 reports, for each optimisation associated to each deflection method (KI=Kinetic Impactor, IBD=Ion Beam Shepherd, $\mathrm{LA}=$ Laser Ablation), the box constraints on the decision variables ToF and $t_{d e f l t}$, the number of agents used by MACS2 to search for the Pareto set (\#agents), and total number of calls to the two objective functions (\#fevals) where one function call evaluates both objectives at the same time.

\begin{tabular}{rllll}
\hline Method & ToF (days) & $t_{\text {defl }}($ days $)$ & \#agents & \#fevals \\
\hline KI & {$[0,1000]$} & {$[0,3662.42]$} & 150 & 14000 \\
IBS & {$[300,2000]$} & {$[0,3662.42]$} & 150 & 3000 \\
LA & {$[300,2000]$} & {$[0,3662.42]$} & 150 & 3000 \\
\hline
\end{tabular}

Table 3: Parameters and box constrains used during the optimisation with MACS2

\subsection{Example with (99942) Apophis and 2011AG5}

As an example of multi-objective optimization, we show here the Pareto fronts we obtained by considering the maximum miss-distance in minimizing the total duration from mission departure to the MOID epoch. The two asteroids considered in this case are a down-scaled version of (99942) Apophis and the actual asteroid 2011AG5 previously considered by NEOSHIELD (2012). In both cases, the construction of the virtual impactor scenarios followed the approached detailed in section 5.2. The results are represented on Fig. 17. Interestingly, note that the Pareto optimum identified in Fig. 17b for a duration of 10 years is remarkably consistent with the maximum value found by evaluating the miss-distance over the entire parameter space in Fig. 3. 


\section{Results and Discussion}

Using the methodology described in the previous section, we computed the maximum miss-distance within 10 years $\left(t_{\mathrm{MOID}}-t_{D}<10\right.$ years $)$ with MPAIDEA and the minimum mission time $\left(t_{\mathrm{MOID}}-t_{D}\right)$ to achieve a miss-distance superior to 2 Earth radii with MACS2.

The results of the maximum miss-distance obtained with MP-AIDEA can be seen in Fig. 18 for the case of a $156 \mathrm{~m}$ asteroid. The Kinetic impactor outperforms the other methods in $78 \%$ of the scenarios. The laser ablation method had the edge in the remaining $22 \%$ of the cases, which corresponded to asteroids with easily accessible orbits from the Earth (low eccentricity, inclination and orbital period close to 1 year). Note that the dots on this plot have a different inclination (coming from the sample distribution in a, e, and i) so that dots with similar semi-major axis and eccentricity may not necessarily produce a similar result on this $2 \mathrm{D}$ plot.

The results of the minimum time to achieve a 2 Earth radii $\left(2 \mathrm{R}_{E}\right)$ deflection can be seen in Fig. 19 for the case of a $156 \mathrm{~m}$ asteroid. In this plot, red points indicate non-feasible deflection solutions within a range of 10 years between departure date and MOID epoch. A total of 84 PHAs can be deflected by the Kinetic Impactor method, against 46 by the Laser Ablation strategy. Note that due to their low performance, the IBS and GT methods were not included in this second analysis. Interestingly, remark again that the KI method performs badly for a subset of virtual Impactor scenario having an orbital period close to 1 year and low eccentricity for which Laser Ablation possesses a superior deflection ability. This complementarity is highlighted if either the Kinetic Impactor or the Laser ablation can be considered. In that case, Fig. 19c shows that $95 \%$ of the PHAs can be deflected. The few asteroids that cannot be deflected in the prescribed time limit have an unfavourable phasing or are nearly tangent to the orbit of the Earth. Indeed, in these case we found that either the Kinetic Impactor or the Laser Ablation were falling short of 2 Earth radii limit although in some cases only by a few $\mathrm{km}$.

\section{Conclusion}

This paper compared four classes of deflection methods, Kinetic Impactor, Ion Beaming, Laser Ablation and Gravity Tractor, applied to a statistically relevant set of deflection scenarios assuming an asteroid mass of $4 \times 10^{9} \mathrm{~kg}$. 
It was demonstrated that the Kinetic Impactor outperforms the other techniques in the majority of the cases. However, detailed investigations reveal that the Kinetic Impactor performs badly for a subset of virtual impactors having an orbital period close to 1 year, a low eccentricity and a low inclination.

For these cases, Laser Ablation offers a superior deflection ability (sometimes by more than one order of magnitude). In all these cases low-cost transfer trajectories provide easy access to the asteroid and the major part of the spacecraft can be allocated to Power and Laser systems. In particular, if either the Kinetic Impactor or the Laser ablation can be considered, up to $95 \%$ of the PHAs can be deflected by over 2 Earth radii within a maximum duration of 10 years between departure date and epoch of the MOID.

Additional interest for these scenarios arises due to the fact that they represent possible targets for future exploration and exploitation missions. Therefore, our results plead for the parallel development of both technologies in the future. To be noted that, in this paper, the optimal transfer for low thrust propulsion was approximated with a spherical shaping algorithm. A better trajectory design might result in an improved performance of all slowpush methods.

Note that, since the deflection distance scales linearly with the the asteroid mass and, as a first approximation, with the spacecraft mass, the authors expect that these choices will not impact the general conclusions of the paper.

Future work will incorporate other interesting methods that have not been considered yet, like the kinetic impactor with low-thrust transfer and electrostatic tractors.

\section{Acknowledgment}

The work in this paper was partially supported by the Marie Curie FP7PEOPLE-2012-ITN Stardust, grant agreement 317185. The authors express their gratitude to Niccolò Gastaldello for implementing the Spherical Shaping algorithm used in this paper as well as Lorenzo Ricciardi and Marilena Di Carlo at the University of Strathclyde for their help in setting the parameters of MACS2 and MP-AIDEA.

\section{References}

Bach, F., 2012. A parametric assessment of the full pha phase space, and deflection feasibility using kinetic impactors. Tech. rep., ESA/ESOC. 
Bombardelli, C., Urrutxua, H., Merino, M., Peláez, J., Ahedo, E., 2013. The ion beam shepherd: A new concept for asteroid deflection. Acta Astronautica 90 (1), 98-102.

Chapman, C. R., 2004. The hazard of near-earth asteroid impacts on earth. Earth and Planetary Science Letters 222 (1), 1-15.

Colombo, C., Vasile, M., Radice, G., 2009. Semi-analytical solution for the optimal low-thrust deflection of near-earth objects. Journal of Guidance, Control, and Dynamics 32 (4), 796-809.

Conway, B., 1997. Optimal low-thrust interception of earth-crossing asteroids. Journal of Guidance Control and Dynamics 20 (5), 995-1002.

Crump, P., Dong, W., Grimshaw, M., Wang, J., Patterson, S., Wise, D., DeFranza, M., Elim, S., Zhang, S., Bougher, M., et al., 2007. 100+ w diode laser bars show $>71 \%$ power conversion from $790 \mathrm{~nm}$ to $1000 \mathrm{~nm}$ and have clear route to $>85 \%$. In: Lasers and Applications in Science and Engineering. International Society for Optics and Photonics, pp. 64560M$64560 \mathrm{M}$.

Di Carlo, M., Vasile, M., Minisci, E., 2015. Multi-population inflationary differential evolution algorithm with adaptive local restart. In: IEEE Congress on Evolutionary Computation. pp. 632-639.

Drachenberg, D., Divliansky, I., Smirnov, V., Venus, G., Glebov, L., 2011. High-power spectral beam combining of fiber lasers with ultra high-spectral density by thermal tuning of volume bragg gratings. In: SPIE LASE. International Society for Optics and Photonics, pp. 79141F-79141F.

Gibbings, A., Vasile, M., Watson, I., Hopkins, J.-M., Burns, D., 2013. Experimental analysis of laser ablated plumes for asteroid deflection and exploitation. Acta Astronautica 90 (1), 85 - 97, NEO Planetary Defense: From Threat to Action.

Honea, E., Afzal, R. S., Savage-Leuchs, M., Gitkind, N., Humphreys, R., Henrie, J., Brar, K., Jander, D., 2013. Spectrally beam combined fiber lasers for high power, efficiency, and brightness. In: SPIE LASE. International Society for Optics and Photonics, pp. 860115-860115. 
Jeong, Y. e., Sahu, J., Payne, D., Nilsson, J., 2004. Ytterbium-doped largecore fiber laser with $1.36 \mathrm{kw}$ continuous-wave output power. Optics Express 12 (25), 6088-6092.

Lu, E. T., Love1, S. G., 2005. Gravity tractor for towing asteroids. Nature (438), 177-178.

McInnes, C., 2007. Near earth object orbit modification using gravitational coupling. Journal of Guidance, Control and Dynamics 30, 870-873.

NEOSHIELD, 2012. D7.5.1: Trade offs of viable alternative mitigation concepts. Tech. rep., ASTRIUM UK.

Novak, D., Vasile, M., 2011. Improved shaping approach to the preliminary design of low-thrust trajectories. Journal of guidance, control, and dynamics 34 (1), 128-147.

Phipps, C., 2011. An alternate treatment of the vapor-plasma transition. International Journal of Aerospace Innovations 3 (1), 45-50.

Phipps, C., Albrecht, G., Friedman, H., Gavel, D., George, E., Murray, J., Ho, C., Priedhorsky, W., Michaelis, M., Reilly, J., 1996. Orion: Clearing near-earth space debris using a 20-kw, 530-nm, earth-based, repetitively pulsed laser. Laser and Particle Beams 14 (01), 1-44.

Phipps, C., Turner, T., Harrison, R., York, G., Osborne, W., Anderson, G., Corlis, X., Haynes, L., Steele, H., Spicochi, K., et al., 1988. Impulse coupling to targets in vacuum by krf, hf, and co2 single-pulse lasers. Journal of Applied Physics 64 (3), 1083-1096.

Phipps, C. R., 2014. L adroit-a spaceborne ultraviolet laser system for space debris clearing. Acta Astronautica 104 (1), 243-255.

Poueyo-Verwaerde, A., Fabbro, R., Deshors, G., De Frutos, A., Orza, J., 1993. Experimental study of laser-induced plasma in welding conditions with continuous co2 laser. Journal of applied physics 74 (9), 5773-5780.

Ricciardi, L. A., Vasile, M., 2015. Improved archiving and search strategies for multi agent collaborative search. In: EUROGEN. 
Rosen, D., Mitteldorf, J., Kothandaraman, G., Pirri, A., Pugh, E., 1982. Coupling of pulsed $0.35-\mu \mathrm{m}$ laser radiation to aluminum alloys. Journal of applied Physics 53 (4), 3190-3200.

Sanchez, P., Colombo, C., Vasile, M., Radice, G., 2009. Multicriteria comparison among several mitigation strategies for dangerous near-earth objects. Journal of Guidance, Control, and Dynamics 32 (1), 121-142.

Tedeschi, W., Remo, J., Schulze, J., Young, R., 1995. Experimental hypervelocity impact effects on simulated planetesimal materials. International Journal of Impact Engineering 17 (4-6), 837-848.

Thiry, N., Vasile, M., Monchieri, E., 2016. Mission and system design for the manipulation of phos with space-borne lasers. In: IEEE Aerospace Conference. pp. 1-13.

Vasile, M., Colombo, C., July 2008. Optimal impact strategies for asteroid deflection. Journal of Guidance, Control, and Dynamics 31 (4).

Vasile, M., Gibbings, A., Massimo, V., Sanchez, J.-P., Yarnoz, D., Eckersley, S., Wayman, A., Branco, J., Burns, D., Hopkins, J.-M., Colombo, C., McInnes, C., 2013. Light Touch2: Effective solutions to asteroid manipulation, SYSNova Challenge Analysis Final Report. Tech. rep., University of Strathclyde.

Vetrisano, M., Cano, J. L., Thiry, N., Tardioli, C., Vasile, M., 2016. Optimal control of a space-borne laser system for a $100 \mathrm{~m}$ asteroid deflection under uncertainties. In: 2016 IEEE Aerospace Conference. IEEE, pp. 1-13.

Vetrisano, M., Thiry, N., Vasile, M., 2015. Detumbling large space debris via laser ablation. In: IEEE Aerospace Conference. pp. 1-10.

Wise, M., Lafleur, J., Saleh, J., 2010. Regression analysis of launch vehicle payload capability for interplanetary missions. In: 61st International Astronomical Congress.

Yamaguchi, K., Yamakawa, H., 2014. Orbital deflection of potentially hazardous asteroids using a coulomb force attractor. Transactions of Japan Society for Aeronautical and Space Sciences 12 (29), 27-33. 
Zuiani, F., Vasile, M., 2015. Extended analytical formulas for the perturbed keplerian motion under a constant control acceleration. Celestial Mechanics and Dynamical Astronomy 121 (3), 275-300.

Zuiani, F., Vasile, M., Gibbings, A., 2012. Evidence-based robust design of deflection actions for near earth objects celestial mechanics and dynamical astronomy. Celestial Mechanics and Dynamical Astronomy 114 (1-2), 107136. 


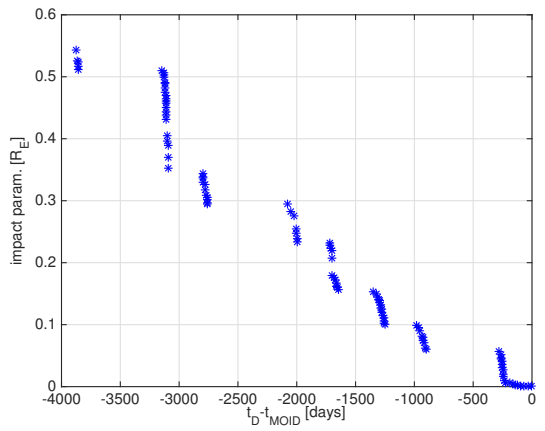

(a) Kinetic Impactor (Apophis)

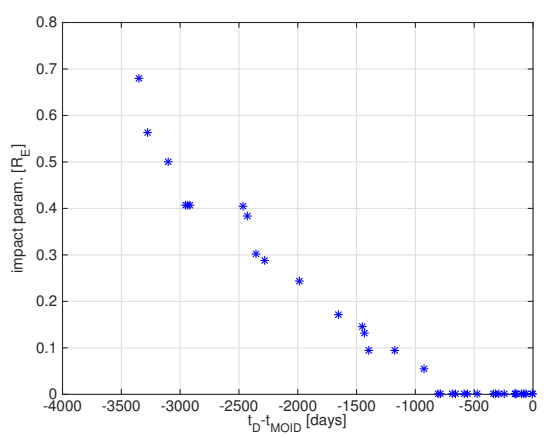

(c) Ion Beam Shepherd (Apophis)

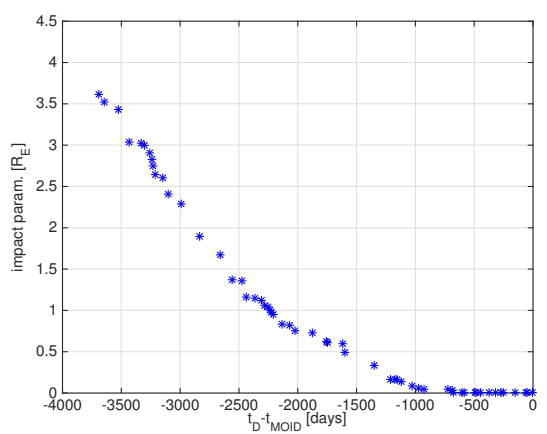

(e) Laser Ablation (Apophis)

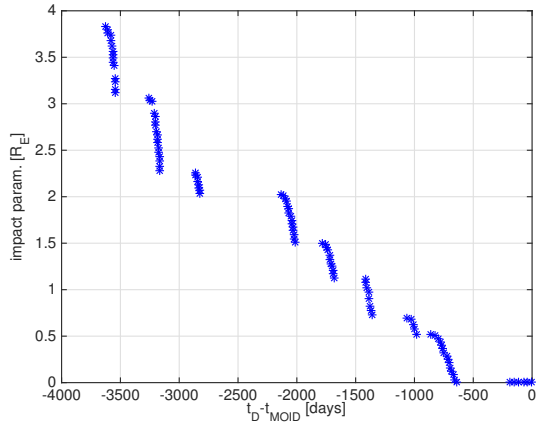

(b) Kinetic Impactor (2011AG5)

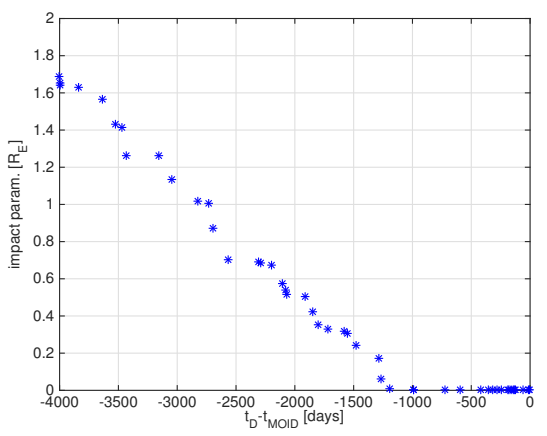

(d) Ion Beam Shepherd (2011AG5)

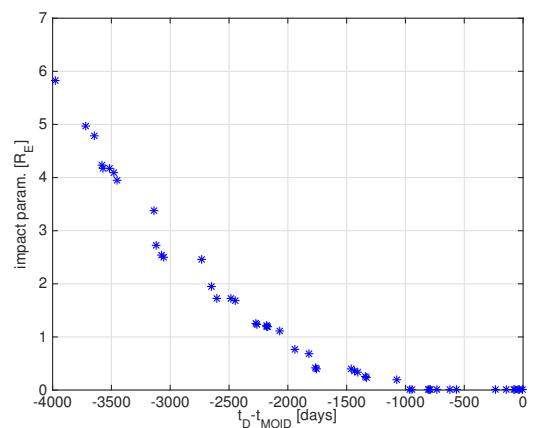

(f) Laser Ablation (2011AG5)

Figure 17: Maximum miss-distance and maximum departure date for the deflection of a $10^{10} \mathrm{~kg}, 212 \mathrm{~m}$ diameter Apophis-like asteroid (left) and a $4 \times 10^{9} \mathrm{~kg}, 156 \mathrm{~m}$ diameter 2011AG5-like asteroid (right) with a S/C launched by Delta 4 heavy 


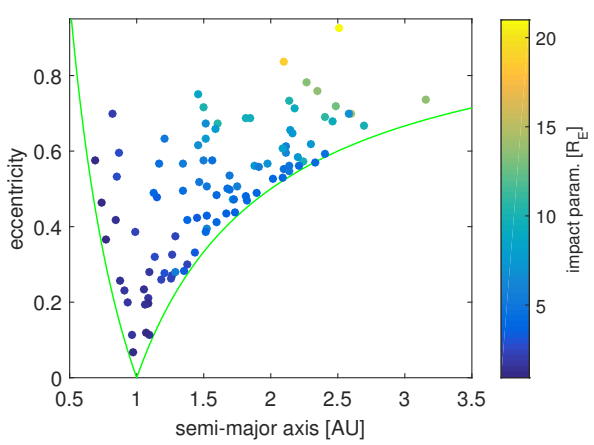

(a) Kinetic Impactor

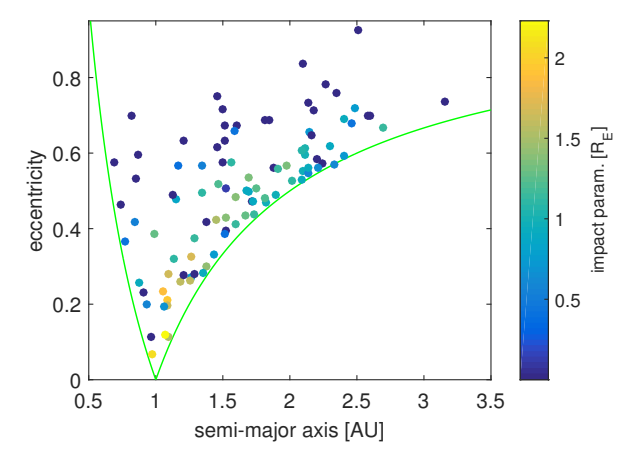

(b) Ion Beam Shepherd

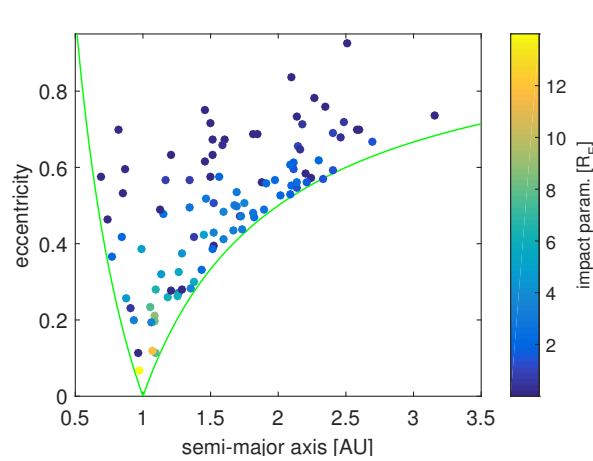

(c) Laser Ablation System

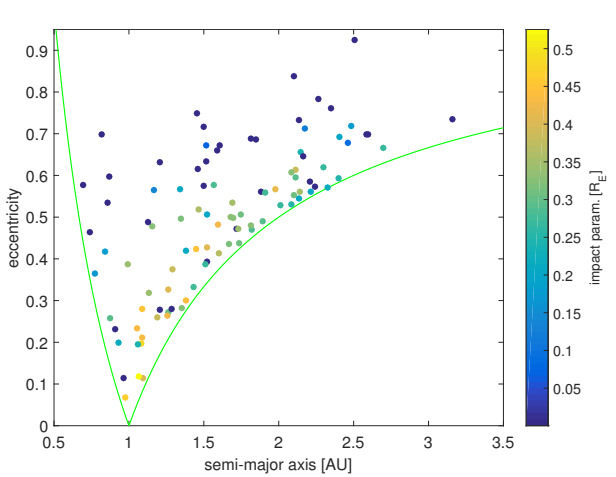

(d) Gravity Tractor

Figure 18: Optimal deflection of a $4 \times 10^{9} \mathrm{~kg}, 156 \mathrm{~m}$ diameter asteroid within 10 years of mission time 


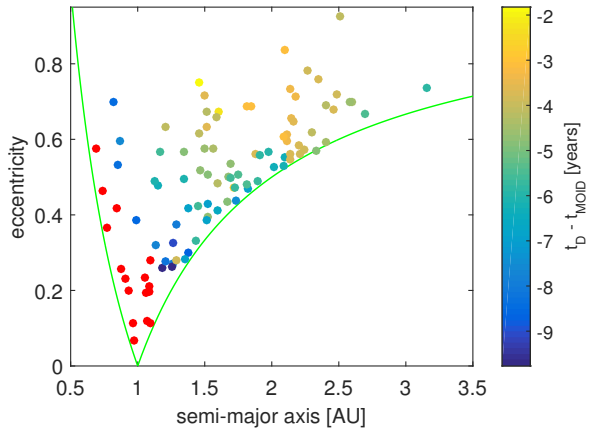

(a) Kinetic Impactor

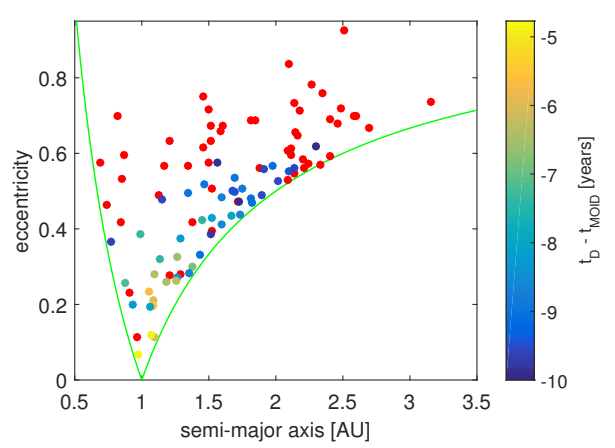

(b) Laser Ablation

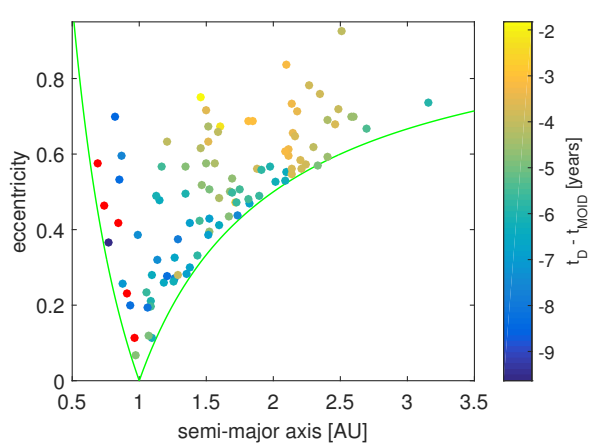

(c) Kinetic Impactor and Laser Ablation

Figure 19: latest departure time for the deflection of a $4 \times 10^{9} \mathrm{~kg}$ asteroid by 2 earth radii with a S/C launched by Delta 4 heavy. Red points indicate unsuccessful missions within 10 years 